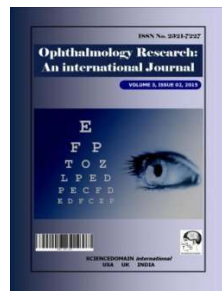

\title{
Post-stroke Visual Impairment: A Systematic Literature Review of Types and Recovery of Visual Conditions
}

\author{
Lauren R. Hepworth ${ }^{1}$, Fiona J. Rowe ${ }^{1 *}$, Marion F. Walker ${ }^{2}$, Janet Rockliffe ${ }^{3}$, \\ Carmel Noonan ${ }^{4}$, Claire Howard ${ }^{5}$ and Jim Currie ${ }^{6}$ \\ ${ }^{1}$ Department of Health Services Research, University of Liverpool, Liverpool L69 3GB, \\ United Kingdom. \\ ${ }^{2}$ Department of Rehabilitation and Ageing, University of Nottingham, Nottingham NG7 2UH, \\ United Kingdom. \\ ${ }^{3}$ Speakability (North West Development Group), 1 Royal Street, London SE1 7LL, United Kingdom. \\ ${ }^{4}$ Department of Ophthalmology, Aintree University Hospital NHS Foundation Trust, L9 7AL, \\ United Kingdom. \\ ${ }^{5}$ Department of Orthoptics, Salford Royal NHS Foundation Trust, Manchester M6 8HD, \\ United Kingdom. \\ ${ }^{6}$ Different Strokes (London South East), 9 Canon Harnett Court, Wolverton Mill, MK12 5NF, \\ United Kingdom.
}

\section{Authors' contributions}

This work was carried out in collaboration between all authors. Author LRH ran searches, identified relevant studies, acted as first review author, extracted data, entered data, provided content expertise and co-wrote the final drafts. Author FJR lead this review, provided methodological expertise, acted as a second review author, carried out analyses, and co-wrote the final drafts. Authors MFW, JR, CN,

$\mathrm{CH}$ and $\mathrm{JC}$ provided additional content expertise, read and commented on final drafts and acted as additional reviewers where there was uncertainty or disagreement. All authors read and approved the 'final manuscript.

\section{Article Information}

DOI: $10.9734 / \mathrm{OR} / 2016 / 21767$

Editor(s):

(1) Yüksel Totan, Department of Ophthalmology, Turgut Özal University, Turkey. Reviewers:

(1) Arturo Solís Herrera, Human Photosynthesis Study Center, Mexico.

(2) Italo Giuffre, Catholic University of Roma, Italy. Complete Peer review History: http://sciencedomain.org/review-history/12294

Review Article

Received $2^{\text {nd }}$ September 2015

Accepted 17 $7^{\text {th }}$ September 2015

Published $16^{\text {th }}$ November 2015 


\section{ABSTRACT}

Aim: The aim of this literature review was to determine the reported incidence and prevalence of visual impairment due to stroke for all visual conditions including central vision loss, visual field loss, eye movement problems and visual perception problems. A further aim was to document the reported rate and extent of recovery of visual conditions post stroke.

Methods: A systematic review of the literature was conducted including all languages and translations obtained. The review covered adult participants (aged 18 years or over) diagnosed with a visual impairment as a direct cause of a stroke. Studies which included mixed populations were included if over $50 \%$ of the participants had a diagnosis of stroke. We searched scholarly online resources and hand searched journals and registers of published, unpublished and ongoing trials. Search terms included a variety of MESH terms and alternatives in relation to stroke and visual conditions. The quality of the evidence was assessed using key reporting guidelines, e.g. STROBE, CONSORT.

Results: Sixty-one studies $(n=25,672)$ were included in the review. Overall prevalence of visual impairment early after stroke was estimated at $65 \%$, ranging from $19 \%$ to $92 \%$. Visual field loss reports ranged from $5.5 \%$ to $57 \%$, ocular motility problems from $22 \%$ to $54 \%$, visual inattention from $14 \%$ to $82 \%$ and reduced central vision reported in up to $70 \%$. Recovery of visual field loss varied between $0 \%$ and $72 \%$, with ocular motility between $7 \%$ and $92 \%$ and visual inattention between $29 \%$ and $78 \%$.

Conclusion: The current literature provides a range of estimates for prevalence of visual impairment after stroke. Visual impairment post stroke is a common problem and has significant relevance to the assessment and care these patients receive. Prospective figures regarding incidence remain unknown.

Keywords: Incidence; prevalence; visual impairment; stroke; recovery; review.

\section{INTRODUCTION}

Types of visual impairment following stroke can be complex including ocular as well as cortical damage [1-6]. Visual impairment can have a wide ranging impact on activities of daily living, independence and quality of life. Links with depression have also been found [7-11]. Many studies provide information on prevalence of various visual conditions from their sample based on cross section and case note observation studies [12-17]. Accurate estimates of prevalence or incidence of visual impairment for stroke survivors remains unknown. Determination of prevalence of visual impairment in a stroke unit is important in order to enable appropriate planning of efficacious referrals to an eye specialist for assessment, treatment and targeted advice $[6,18,19]$.

The aim of this systematic literature review was to provide a comprehensive synthesis and exploration of reported evidence relating to visual problems after stroke with specific attention to incidence and prevalence.

\subsection{Visual Impairment Definitions}

Visual impairment is a deficit of visual function and includes abnormalities of peripheral vision, central vision, eye movements and a variety of perception problems $[1,3,4,20]$.

Visual field loss is loss of a section of the field of vision and can either be central or peripheral. Following stroke visual field loss is frequently homonymous, with a loss in the same half of the visual field of both eyes. The types of visual field loss can include, hemianopia, quadrantanopia, constriction and scotomas [20,21]. It is also possible to have a loss of the central area of vision.

There are a wide range of ocular motility problems which can occur as a result of stroke including strabismus, cranial nerve palsies, gaze palsies, vergence abnormalities and nystagmus [22]. Strabismus is the misalignment of the eyes, which can be longstanding from childhood or occur as a result of an insult to the extra-ocular muscles or the cranial nerves supplying them. Eye movement palsies or pareses following stroke can include cranial nerve palsy, horizontal gaze palsy and/or vertical gaze palsy. Nystagmus is a continuous oscillatory movement of the eyes and is frequently associated in which both eyes move symmetrically. It may occur in every position of gaze or only be present in certain gaze positions. A further consideration is 
that patients commonly have multiple defects concurrently [23].

There are a number of different perceptual problems which can occur after stroke. The most recognised is visual inattention/neglect, in which the individual does not respond or attend to visual stimuli on the affected side. Other perceptual problems are also reported such as agnosia, visual hallucinations and image movement problems [24].

\section{METHODS}

We conducted an integrative review, aiming to bring together all evidence relating to incidence, prevalence and recovery from stroke-related visual problems. The review observed and is reported according to the PRISMA guidelines (Appendix 1). This review was not registered with PROSPERO [25].

\subsection{Inclusion Criteria for Considering Studies for This Review}

\subsubsection{Types of studies}

The following types of studies were included: randomised controlled trials, controlled trials, prospective and retrospective cohort studies and observational studies. Case reports and casecontrolled studies were excluded, as they specifically look at selected cases and are therefore unable to report incidence or prevalence. All languages were included and translations obtained when necessary.

\subsubsection{Types of participants}

We included studies of adult participants (aged 18 years or over) diagnosed with a visual impairment as a direct result of a stroke. Studies which included mixed populations were included if over $50 \%$ of the participants had a diagnosis of stroke and data were available for this subgroup.

\subsubsection{Types of outcome and data}

We defined incidence as the number of new cases of any visual condition occurring during a certain period in a stroke survivor population. We defined prevalence as the number of cases of any visual condition present in a stroke survivor population at a certain time. We defined a measure of recovery as being present if prevalence figures were available at more than one time point post stroke. The visual impairments included are defined below.

\subsection{Visual Impairment Definitions}

Visual impairment is a deficit of visual function and includes abnormalities of peripheral vision, central vision, eye movements and a variety of perception problems $[1,3,4,20]$.

Visual field loss is loss of a section of the field of vision and can either be central or peripheral. Following stroke visual field loss is frequently homonymous, with a loss in the same half of the visual field of both eyes. The types of visual field loss can include, hemianopia, quadrantanopia, constriction and scotomas [20,21]. It is also possible to have a loss of the central area of vision.

There are a wide range of ocular motility problems which can occur as a result of stroke including strabismus, cranial nerve palsies, gaze palsies, vergence abnormalities and nystagmus [22]. Strabismus is the misalignment of the eyes, which can be longstanding from childhood or occur as a result of an insult to the extra-ocular muscles or the cranial nerves supplying them. Eye movement palsies or paresis following stroke can include cranial nerve palsy, horizontal gaze palsy and/or vertical gaze palsy. Nystagmus is a continuous oscillatory movement of the eyes and is frequently associated in which both eyes move symmetrically. It may occur in every position of gaze or only be present in certain gaze positions. A further consideration is that patients commonly have multiple defects concurrently [23].

There are a number of different perceptual problems which can occur after stroke. The most recognised is visual inattention/neglect, in which the individual does not respond or attend to visual stimuli on the affected side. Other perceptual problems are also reported such as agnosia, visual hallucinations and image movement problems [24].

\subsection{Search Methods for Identification of Studies}

We used systematic strategies to search key electronic databases and contacted known individuals conducting research in stroke and visual impairment. We searched Cochrane registers and electronic bibliographic databases (Appendix 2). In an effort to identify further published, unpublished and ongoing trials, we searched registers of ongoing trials, handsearched journals and conference transactions, 
performed citation tracking using Web of Science Cited Reference Search for all included studies, searched the reference lists of included trials and review articles about vision after acquired brain injury and contacted experts in the field (including authors of included trials, and excluded studies identified as possible preliminary or pilot work). Search terms included a comprehensive range of $\mathrm{MeSH}$ terms and alternatives in relation to stroke and visual conditions (Appendix 2).

\subsection{Selection of Studies}

The titles and abstracts identified from the search were independently screened by two authors (FR, LH) using the pre-stated inclusion criteria. The full papers of any studies considered potentially relevant were then considered and the selection criteria applied independently by two reviewers (FR, LH). In the case of disagreement for inclusion of studies, an option was available to obtain a third author opinion (CN).

\subsection{Data Extraction}

A pre-designed data extraction form was used which gathered information on sample size, study design, assessments undertaken, visual conditions reported, timing of assessment and population type. Data was extracted and documented by one researcher (LH) and verified by another (FR).

\subsection{Data Analysis}

Due to the heterogeneous nature of the studies, a narrative analysis was undertaken. The exception to this was a calculation to estimate the prevalence of overall visual impairment following stroke. Strict criteria of only studies using consecutive recruitment from a stroke population and reporting an overall prevalence for visual impairment were used for the mean prevalence calculation.

\subsection{Quality Assessment}

To assess the quality of the studies included in this review, two checklists were considered relevant to the study designs in our inclusion criteria: the STROBE (Strengthening the Reporting of Observational Studies in Epidemiology) checklist [26,27]. The checklist was adapted as the original was designed to assess the quality of reporting rather than the potential for bias within a study. There is currently no 'gold standard' quality assessment tool for observational studies [28]. The STROBE Statement covers 22 items covering the whole of the articles from introduction, method, results and discussion, which are important to consider when assessing the quality of observation studies (including cohort, case-control and crosssectional studies). The adapted version used in this review included 18 items; only the information which is pertinent to quality appraisal of the studies was included. Using Boyle's recommendations for the evaluation of prevalence studies, the items exclude which were not considered relevant information, such as the title, abstract, background, setting and funding [29].

\section{RESULTS OF THE SEARCH}

The search results are outlined in Appendix 3. Sixty-four articles $(26,321$ participants) were included. Of the 64 included studies, none of which were RCTs, 52 were prospective observational studies and 12 were retrospective analyses. Consequently quality of study was assessed using the STROBE checklist. Although none of the studies were RCTs, one study was a retrospective analysis of data from an RCT archive [30]. Studies excluded from this review are outlined in a Appendix 4. Quality appraisal using the adapted STROBE checklist is outlined in a Appendix 5.

Seven of the studies (14,573 participants) reported on overall visual impairment. Nineteen of the studies $(17,924$ participants) reported on visual field defects; 22 of the studies (4330 participants) reported on ocular alignment and motility defects; nine of the studies (2097 participants) reported on central vision problems; and 13 of the studies (2885 participants) reported on types of perceptual visual deficits following stroke (including visual neglect/inattention, visual hallucinations, agnosia and reduced stereopsis). Several studies reported on two or more of these categories.

None of the studies included had a specific primary aim to calculate either prevalence or incidence of visual impairment following stroke. Fifty five studies were studies specifically investigated visual impairment following stroke, this included studies looking at specific visual problems such as visual inattention. The remaining 16 studies investigated symptoms and signs of stroke, which included reported visual impairment. 


\section{QUALITY OF THE EVIDENCE}

Three paper reported $100 \%$ of the items requested by the adapted STROBE checklist [31]. Sixteen papers reported $90 \%$ or more of the requested items, 51 papers reported $75 \%$ or more. Sixty-one reported $50 \%$ or more and three papers failed to reach $50 \%$, achieving $17 \%$, $33 \%$ and $39 \%$ [32-34]. Only $36 \%$ of papers reported limitations of their studies. Results from all papers were reported and the individual results for each paper are outlined in a Appendix 5.

\section{PREVALENCE AND INCIDENCE}

\subsection{Visual Impairment}

Our search of the literature did not reveal any studies that specifically aimed to assess the incidence of visual impairment following stroke. We identified a number of studies that report an overall figure of prevalence for visual impairment. All these studies, however, were judged to have limitations relating to the methods of recruitment or assessment. Thus a calculation of incidence was not possible and estimates are calculated for prevalence.

Three prospective studies of stroke populations $(n=709)$ report an average prevalence of visual impairment post stroke of $65 \%$ ranging from 62 $71 \%$ (Table 1) $[32,33,35]$. These studies evaluated a general stroke population including medical and orthoptic assessments undertaken during the acute stroke phase within one week of onset to three months post stroke onset. Further to these three studies of general stroke populations, one prospective study $(n=915)$ recruited a sub population of stroke survivors with suspected visual impairment who received full orthoptic assessment, typically within three weeks of stroke onset [6]. They reported a prevalence of $92 \%$ visual impairment. It is unknown what was missed from the general stroke population as not all individuals can report visual symptoms and referrals were evaluated to be more accurate when visual symptoms were taken into consideration in addition to ocular signs in comparison to ocular signs alone [36]. Ali et al., analysed results from a database for stroke survivors recruited to a variety of strokerelated clinical trials and reported a baseline prevalence of $60 \%$ visual impairment [30]. This cohort would typically include those who are able and willing to participate in a clinical trial and are therefore, not representative of the whole population, for example individuals with cognitive impairment and aphasia are less likely to be recruited [37].

Three studies $(n=13,541)$ used a stroke assessment tool (NIHSS \pm status questionnaire) which only partly assesses visual function $[30,31,38]$. The National Institute of Health Stroke Scale (NIHSS) is an assessment tool that only assesses for the presence of visual field loss and horizontal gaze problems [39]. Thus it is not a full assessment of the possible visual problems which can manifest as a result of stroke. It can therefore be argued that the numbers presented by these studies are not a true measure of overall incidence of visual impairment following stroke. In addition to the NIHSS, the Questionnaire for Verifying Strokefree Status (QVSFS) was used. However this questionnaire only asks the patient about painless complete or partial vision loss [40]. The range of overall incidence of visual problems was $19-25.9 \%$ from these studies which was considerably less than studies with more comprehensive vision assessment methods.

\subsection{Visual Field Loss}

The reported prevalence of visual field loss after stroke varies considerably in the literature from $5.5 \%$ to $57 \%$ (Table 2 ) and most probably due to its dependence on the type and affected area of a stroke, inclusion criteria and the timing of assessments and the method of testing used [41-44].

Seven studies $(n=1210)$ recruited stroke patients consecutively either as they were admitted to hospital acute stroke units or rehabilitation wards. Assessment of visual fields by confrontation and/or perimetry on admission after stroke onset detected visual field loss in up to $57 \%[32,33,41,45-48]$. The mean prevalence of visual field loss after stroke was calculated as $31 \%[32,33,41,45-48]$. These studies typically assessed patients in the acute phase with homonymous hemianopia or quadrantanopia defects most frequently detected.

In addition to the above studies, seven prospective studies $(n=15,388)$ of stroke subpopulations report prevalence of visual field loss [21,30,43,49-51]. These sub-populations typically include only stroke survivors with hemianopic or quadrantanopic field loss or with suspected visual impairment of any type, or do not recruit consecutively. Thus reported prevalence is not representative of the full stroke population. 
Prevalence of visual field loss has been described based on symptom reporting by patients in four studies $(n=1362)$ ranging from 14.6 to $22.7 \%$ [42,52-54]. These reports are considerably lower and likely reflecting the poor reliability of detection by patient reported symptoms. In addition to those formally diagnosed with visual field loss following stroke, it is important to consider how many patients are unaware of their visual loss. Celesia et al. conducted a prospective observation study $(n=32)$ to investigate the presence of hemianopic anosognosia [54]. From a sample of thirty two patients with homonymous visual field loss, $62 \%$ were unaware of their visual deficit. In a recent paper it was reported that only $45 \%$ of participants with visual field loss reported symptoms of the visual field loss [36]. It is important to note that not all patients had isolated visual field loss. Multiple visual impairments caused by stroke were reported such as visual acuity loss, eye movement abnormalities and perceptual difficulties. This discrepancy between those who do not complain of symptoms and have a diagnosis of visual field loss may highlight an under estimation in the incidence in this and other studies.

For studies whose population samples have solely included patients with visual field loss post stroke, it is not possible to establish prevalence. However, several of these studies have shown almost equal numbers suffering right or left defects $[34,44,55,56]$.

\subsection{Ocular Motility/Strabismus}

Three prospective studies $(n=1262)$ reported an average prevalence of all ocular motility problems as $33 \%$ (Table 3 ) with a range from $22 \%$ to $54 \%$, [18,35,57]. Assessments were usually within the acute period and two studies used detailed orthoptic evaluation of eye movements and binocular vision [18,35]. Methods of ocular motility assessment are important to the accuracy of identification of eye movement abnormalities to ensure full detection of deficits in various gaze positions.

\subsubsection{Eye alignment}

Strabismus may occur as an isolated finding or in association with ocular motility problems and is reported in $16.5 \%$ to $52 \%$ of stroke survivors recruited to three prospective observation studies $(n=626)$, with an average prevalence of $38 \%$ $[32,35,58]$. These studies used validated orthoptic assessments to detect presence of strabismus, increasing their accuracy of detection. In a sub-population prospective multicentre observational study, $19 \%$ of the sample were identified with strabismus [23]. Pre-existing strabismus was acknowledged in $2.5 \%$, thus $16.5 \%$ were considered to be a direct result of stroke. The cause of the strabismus in $70 \%$ of cases was an ocular motility defect. Only $36 \%$ were symptomatic with diplopia, which highlights an issue in relying purely on symptoms alone. This study has a risk of under-estimating the prevalence, as the sample is not representative of the whole stroke population.

Diplopia is reported as a symptom in many papers which is a result of a misalignment of the eyes and a disruption of binocular vision. Other studies have highlighted the discrepancy between patients who do or do not report diplopia in the presence of strabismus or ocular motility defects. There is a risk that a proportion is not captured, if the symptom of diplopia is relied upon to identify ocular motility defects. The majority of studies reporting the incidence of diplopia limit recruitment to include strokes affecting specific areas of the brain $[43,59,60]$, are retrospective $[42,53]$ or required informed consent [61]. These studies cannot be generalised to the whole stroke population and also carry a risk of under estimating the true prevalence of strabismus.

\subsubsection{Eye movement palsy}

Seven studies $(n=2783)$ report figures for gaze palsies including horizontal and/or vertical gaze positions and have a mean prevalence following stroke of $26 \%$ (range 18-44\%) [22,32,35,43, $57,62,63]$. These defects may occur in isolation or in conjunction with other visual problems, and are the most common of all ocular motility abnormalities [22,57]. Horizontal gaze palsies are more prevalent than vertical and complete palsies more prevalence than partial $[22,32,35,63]$.

Cranial nerve palsies affecting the ocular motor muscles include third, fourth and sixth nerves with a mean post-stroke prevalence of $16 \%$ (range 3 to $39 \%)$ from three studies $(n=2329)$ $[18,32,43,57]$. Third nerve and sixth nerve palsies are reported as being more prevalent than fourth nerve palsies in these stroke populations $[18,32,64]$. Where ocular movement assessment only tests horizontal gaze (such as with the NIHSS screening tool) the 
identification of all ocular cranial nerve palsies is limited. It is likely that more subtle nerve palsies and those involving the vertical muscles may be missed.

\section{$\underline{\text { 5.3.3 Nystagmus }}$}

Following stroke, nystagmus is reported in an average of $11 \%$ (range 4 to $48 \%$ ) in three studies $(n=438) \quad[35,62,65]$. In most prospective and retrospective studies reporting nystagmus, the specific types of nystagmus are not reported. This, in addition to lack of information regarding the method of assessment, makes it difficult to assess if the more subtle types, or nystagmus not present in primary position, have been missed. These factors increase the risk of an underestimation of prevalence. When reported, common types of acquired nystagmus are gaze evoked, multi-vector and upbeat [66]. The studies described to date, frequently report when the stroke has affected the posterior circulation, including the cerebellum [42,60,67,68]. No studies have reported the prevalence of nystagmus in anterior circulation strokes in isolation. It is, therefore not possible to estimate the proportion of cases which are potentially missed by restricting populations to posterior circulation strokes only.

\subsubsection{Vergence}

Clisby $(n=140)$ reported $55 \%$ of patients to have reduced convergence and/or stereopsis [32]. Rowe et al. $(n=243)$ reported reduced convergence from the initial ten month data set of the Vision in Stroke (VIS) study [69]. Using the gold standard 'normal' attainment for convergence of $6 \mathrm{~cm}, 54 \%$ were judged to have reduced convergence. However, they also reported that $26 \%$ had convergence reduced less than $10 \mathrm{~cm}$, which could be judged to be a more appropriate standard for an older group of patients. Siong et al. reported $21 \%$ of the recruited population to have convergence reduced less than $15 \mathrm{~cm}$ [61].

\subsection{Visual Acuity and Central Vision Deficit}

Clinical assessment of visual acuity has been used to identify those with reduced vision and up to $70 \%$ of stroke survivors (Table 4 ) have been noted to have poor central vision $[32,36,64,70]$. The mean prevalence of reduced visual acuity post-stroke was calculated from three studies $(n=270)$ as $53 \%[32,64,70]$. Methods include visual acuity assessment at near, a 3 or 6 metre distance. Further retrospective studies $(n=447)$ provide information on the prevalence of patients reporting symptoms associated with a reduction of visual acuity $[42,53]$. A key issue identified by three studies $(n=1045)$ related to patient glasses $[36,64,70]$. These were frequently reported as missing, or the glasses present were dirty, broken or the wrong prescription.

An important component of central visual function is contrast sensitivity, the reduction of which can deform image perception. Contrast sensitivity function has been reported to be abnormal in $62 \%$ of stroke patients $(n=16)$ [71]. Different areas of the spectrum are impaired depending on the lesion site. For example, participants with parietal and temporal lesions have been reported to have reduced detection of low spatial frequencies whereas those with occipital and occipito-temporal lesions had difficulty with medium to high spatial frequencies [71]. Furthermore, reduced contrast sensitivity in stroke survivors, particularly those with severe functional difficulties, has been found to be associated with reduced activities of daily living [72].

Central vision is key to activities such as reading. However, reading difficulties may be caused by a wide range of visual impairments in addition to reduced visual acuity. Rowe et al. $(n=915)$ reported difficulties with reading occurred in $19.3 \%$ of the sample [19]. The three largest associations with reading difficulties were visual field loss $(61.6 \%$, the majority of which were complete homonymous hemianopia), reduced convergence of less than $6 \mathrm{~cm}(45.8 \%)$ and saccadic abnormalities (45.0\%). Other visual impairments associated with reading difficulties included reduced visual acuity (22.5\%), perceptual deficits $(22 \%)$, including $16.5 \%$ with visual inattention, nystagmus (12.4\%) and diplopia (8.5\%).

\subsection{Visual Perception Abnormalities}

The commonest form of visual perception disorder following stroke is visual neglect or inattention. The literature reporting the prevalence of visual neglect/inattention can be difficult to interpret. Often the different types of inattention (e.g. auditory, visual, and spatial) are not separated, so it is not always possible to isolate visual inattention. 
Table 1. Overall visual impairment prevalence

\begin{tabular}{|c|c|c|c|c|c|c|c|}
\hline Study & Design & Population & $\begin{array}{l}\text { Time of vision } \\
\text { assessment }\end{array}$ & $\begin{array}{l}\text { Sample size } \\
(n=)\end{array}$ & $\begin{array}{l}\text { Prevalence of } \\
\text { visual issue (\%) }\end{array}$ & $\begin{array}{l}\text { Co-existent } \\
\text { ocular condition }\end{array}$ & $\begin{array}{l}\text { Method of visual } \\
\text { assessment }\end{array}$ \\
\hline $\begin{array}{l}\text { 1974; Isaeff } \\
\text { et al. [33] }\end{array}$ & $\begin{array}{l}\text { Prospective } \\
\text { observation }\end{array}$ & General stroke & $\begin{array}{l}\text { Median within } 3 \\
\text { months of onset }\end{array}$ & 322 & 62 & Yes & Medical \\
\hline $\begin{array}{l}\text { 1987; } \\
\text { Freeman \& } \\
\text { Rudge [35] }\end{array}$ & $\begin{array}{l}\text { Prospective } \\
\text { observation }\end{array}$ & General stroke & $\begin{array}{l}\text { Median within } 1 \text { week } \\
\text { of onset }\end{array}$ & 247 & 63 & Yes & $\begin{array}{l}\text { Medical } \\
\text { Orthoptic }\end{array}$ \\
\hline $\begin{array}{l}\text { 1995; Clisby } \\
\text { [32] }\end{array}$ & $\begin{array}{l}\text { Prospective } \\
\text { observation }\end{array}$ & General stroke & $\begin{array}{l}\text { Acute period on } \\
\text { stroke unit }\end{array}$ & 140 & 71 & Yes & Orthoptic \\
\hline $\begin{array}{l}\text { 2007; Barrett } \\
\text { et al. [38] }\end{array}$ & $\begin{array}{l}\text { Prospective } \\
\text { observation }\end{array}$ & General stroke & Unknown & 505 & 19 & Unknown & $\begin{array}{l}\text { NIHSS and } \\
\text { Questionnaire for } \\
\text { verifying stroke- } \\
\text { free status }\end{array}$ \\
\hline $\begin{array}{l}\text { 2009; Rowe } \\
\text { et al. [6] }\end{array}$ & $\begin{array}{l}\text { Prospective } \\
\text { observation }\end{array}$ & $\begin{array}{l}\text { Stroke survivors } \\
\text { with suspected } \\
\text { visual issues }\end{array}$ & $\begin{array}{l}\text { Median within } 3 \\
\text { weeks of onset }\end{array}$ & 323 & 92 & Yes & Orthoptic \\
\hline $\begin{array}{l}\text { 2013; } \\
\text { Ali et al. [30] }\end{array}$ & Trial data & Acute stroke & $\begin{array}{l}\text { Median within } 1 \text { week } \\
\text { of stroke onset }\end{array}$ & 11900 & 60 & Unknown & NIHSS \\
\hline $\begin{array}{l}\text { 2010; Gall } \\
\text { et al. [31] }\end{array}$ & Retrospective & General stroke & Unknown & 1136 & $\begin{array}{l}25.9 \\
23-\text { male } \\
\text { 29-female } \\
\end{array}$ & Unknown & NIHSS \\
\hline
\end{tabular}

Table 2. Visual field loss prevalence

\begin{tabular}{|c|c|c|c|c|c|c|c|}
\hline Study & Design & Population & $\begin{array}{l}\text { Time of vision } \\
\text { assessment }\end{array}$ & $\begin{array}{l}\text { Sample size } \\
(n=)\end{array}$ & $\begin{array}{l}\text { Prevalence of visual } \\
\text { issue (\%) }\end{array}$ & $\begin{array}{l}\text { Co-existent } \\
\text { ocular } \\
\text { condition }\end{array}$ & $\begin{array}{l}\text { Method of visual } \\
\text { field assessment }\end{array}$ \\
\hline $\begin{array}{l}\text { 1973; } \\
\text { Haerer } \\
\text { et al. [47] }\end{array}$ & $\begin{array}{l}\text { Prospective } \\
\text { observation }\end{array}$ & General stroke & Unknown & 265 & $\begin{array}{l}25 \text { - homonymous } \\
\text { hemianopia/ } \\
\text { quadrantanopia }\end{array}$ & Unknown & Confrontation \\
\hline $\begin{array}{l}\text { 1974; } \\
\text { Isaeff } \\
\text { et al. [33] }\end{array}$ & $\begin{array}{l}\text { Prospective } \\
\text { observation }\end{array}$ & General stroke & $\begin{array}{l}\text { Median within } 3 \\
\text { months of onset }\end{array}$ & 322 & 17 - visual field loss & $\begin{array}{l}\text { Ocular } \\
\text { pathology }\end{array}$ & Confrontation \\
\hline $\begin{array}{l}\text { 1989; } \\
\text { Gray }\end{array}$ & $\begin{array}{l}\text { Prospective } \\
\text { observation }\end{array}$ & General stroke & $\begin{array}{l}\text { Followed every } 24 \\
\text { hours for } 4 \text { days }\end{array}$ & 174 & $\begin{array}{l}56.9-\text { homonymous } \\
\text { hemianopia }\end{array}$ & $\begin{array}{l}\text { Ocular } \\
\text { pathology }\end{array}$ & Confrontation \\
\hline
\end{tabular}


Hepworth et al.; OR, 5(1): 1-43, 2016; Article no.OR.21767

\begin{tabular}{|c|c|c|c|c|c|c|c|}
\hline Study & Design & Population & $\begin{array}{l}\text { Time of vision } \\
\text { assessment }\end{array}$ & $\begin{array}{l}\text { Sample size } \\
(n=)\end{array}$ & $\begin{array}{l}\text { Prevalence of visual } \\
\text { issue (\%) }\end{array}$ & $\begin{array}{l}\text { Co-existent } \\
\text { ocular } \\
\text { condition }\end{array}$ & $\begin{array}{l}\text { Method of visual } \\
\text { field assessment }\end{array}$ \\
\hline et al. [41] & & & and max to 28 days & & $\begin{array}{l}46.6 \text { - hemianopia } \\
10.3 \text { - quadrantanopia }\end{array}$ & & \\
\hline $\begin{array}{l}\text { 1993; } \\
\text { Benedetti } \\
\text { et al. [48] }\end{array}$ & $\begin{array}{l}\text { Prospective } \\
\text { observation }\end{array}$ & General stroke & $\begin{array}{l}\text { Median within } 48 \\
\text { hours of admission }\end{array}$ & 94 & $\begin{array}{l}19.1 \text { - homonymous } \\
\text { hemianopia }\end{array}$ & Unknown & Unknown \\
\hline $\begin{array}{l}\text { 1995; } \\
\text { Clisby [32] }\end{array}$ & $\begin{array}{l}\text { Prospective } \\
\text { observation }\end{array}$ & General stroke & $\begin{array}{l}\text { Acute period on } \\
\text { stroke unit }\end{array}$ & 140 & 47 - visual field loss & $\begin{array}{l}\text { Ocular } \\
\text { pathology }\end{array}$ & $\begin{array}{l}\text { Confrontation } \\
\text { Campimetry }\end{array}$ \\
\hline $\begin{array}{l}\text { 1997; } \\
\text { Agrell } \\
\text { et al. [45] }\end{array}$ & $\begin{array}{l}\text { Prospective } \\
\text { observation }\end{array}$ & General stroke & $\begin{array}{l}\text { Median within } 3 \\
\text { months of onset }\end{array}$ & 67 & $\begin{array}{l}30-\text { homonymous } \\
\text { hemianopia }\end{array}$ & $\begin{array}{l}\text { Visual } \\
\text { inattention }\end{array}$ & Confrontation \\
\hline $\begin{array}{l}\text { 1997; } \\
\text { Celesia } \\
\text { et al. [54] }\end{array}$ & $\begin{array}{l}\text { Prospective } \\
\text { observation }\end{array}$ & $\begin{array}{l}\text { Stroke survivors } \\
\text { with hemianopia }\end{array}$ & $\begin{array}{l}\text { Median within } 24 \\
\text { hours of onset }\end{array}$ & 32 & $\begin{array}{l}100-\text { homonymous } \\
\text { hemianopia } \\
62 \text { - asymptomatic }\end{array}$ & Unknown & Kinetic perimetry \\
\hline $\begin{array}{l}2000 ; \\
\text { Lotery } \\
\text { et al. [64] }\end{array}$ & $\begin{array}{l}\text { Prospective } \\
\text { observation }\end{array}$ & General stroke & $\begin{array}{l}\text { Median within } 3 \\
\text { months of onset }\end{array}$ & 77 & $\begin{array}{l}19.5-\text { visual field loss } \\
3 / 4 \text { hemianopia }\end{array}$ & $\begin{array}{l}\text { Ocular } \\
\text { pathology }\end{array}$ & Unknown \\
\hline $\begin{array}{l}2001 ; \\
\text { Cassidy et } \\
\text { al. [46] }\end{array}$ & $\begin{array}{l}\text { Prospective } \\
\text { observation }\end{array}$ & General stroke & $\begin{array}{l}\text { Median within } 3 \\
\text { months of onset }\end{array}$ & 148 & 50.6 - visual field loss & $\begin{array}{l}\text { Ocular } \\
\text { pathology }\end{array}$ & $\begin{array}{l}\text { Confrontation } \\
\text { Perimetry }\end{array}$ \\
\hline $\begin{array}{l}\text { 2007; } \\
\text { Townsend } \\
\text { et al. [51] }\end{array}$ & $\begin{array}{l}\text { Prospective } \\
\text { observation }\end{array}$ & $\begin{array}{l}\text { General stroke } \\
\text { excluding } \\
\text { receptive aphasia } \\
\text { and cognitive } \\
\text { impairment }\end{array}$ & $\begin{array}{l}\text { Within } 9 \text { months of } \\
\text { onset }\end{array}$ & 61 & $\begin{array}{l}16-\text { homonymous } \\
\text { hemianopia }\end{array}$ & Unknown & Static perimetry \\
\hline $\begin{array}{l}\text { 2009; } \\
\text { Rowe } \\
\text { et al. [6] }\end{array}$ & $\begin{array}{l}\text { Prospective } \\
\text { observation }\end{array}$ & $\begin{array}{l}\text { Stroke survivors } \\
\text { with suspected } \\
\text { visual issues }\end{array}$ & $\begin{array}{l}\text { Median within } 3 \\
\text { weeks of onset }\end{array}$ & 915 & $\begin{array}{l}49.5-\text { visual field loss } \\
2 / 3 \text { hemianopia } \\
1 / 2 \text { asymptomatic }\end{array}$ & $\begin{array}{l}\text { Ocular } \\
\text { pathology } \\
\text { Visual } \\
\text { inattention }\end{array}$ & $\begin{array}{l}\text { Confrontation } \\
\text { Kinetic perimetry } \\
\text { Static perimetry }\end{array}$ \\
\hline $\begin{array}{l}\text { 2012; Tao } \\
\text { et al. [43] }\end{array}$ & $\begin{array}{l}\text { Prospective } \\
\text { observation }\end{array}$ & $\begin{array}{l}\text { General stroke: } \\
\text { anterior vs } \\
\text { posterior } \\
\text { circulation }\end{array}$ & $\begin{array}{l}\text { Median within } 3 \\
\text { months of onset }\end{array}$ & 1174 & $\begin{array}{l}6.9 \text { - visual field loss } \\
\text { Hemianopia: } \\
4.3 \text { - posterior circulation } \\
1.3 \text { - anterior circulation } \\
\text { Quadrantanopia: } 1.3 \text { - } \\
\text { posterior circulation }\end{array}$ & Unknown & $\begin{array}{l}\text { NIHSS } \\
\text { Confrontation }\end{array}$ \\
\hline
\end{tabular}


Hepworth et al.; OR, 5(1): 1-43, 2016; Article no.OR.21767

\begin{tabular}{|c|c|c|c|c|c|c|c|}
\hline Study & Design & Population & $\begin{array}{l}\text { Time of vision } \\
\text { assessment }\end{array}$ & $\begin{array}{l}\text { Sample size } \\
(n=)\end{array}$ & $\begin{array}{l}\text { Prevalence of visual } \\
\text { issue (\%) }\end{array}$ & $\begin{array}{l}\text { Co-existent } \\
\text { ocular } \\
\text { condition }\end{array}$ & $\begin{array}{l}\text { Method of visual } \\
\text { field assessment }\end{array}$ \\
\hline $\begin{array}{l}\text { 2013; Ali } \\
\text { et al. [30] }\end{array}$ & $\begin{array}{l}\text { Prospective trial } \\
\text { data }\end{array}$ & General stroke & $\begin{array}{l}\text { Median within } 1 \\
\text { week of stroke } \\
\text { onset }\end{array}$ & 11900 & $\begin{array}{l}51 \text { - visual field loss: } \\
\text { majority hemianopia }\end{array}$ & Unknown & $\begin{array}{l}\text { NIHSS } \\
\text { Confrontation }\end{array}$ \\
\hline $\begin{array}{l}2013 ; \\
\text { Rowe et } \\
\text { al. [21] }\end{array}$ & Prospective & $\begin{array}{l}\text { Stroke survivors } \\
\text { with suspected } \\
\text { visual impairment }\end{array}$ & $\begin{array}{l}\text { Variable over } 2 \\
\text { weeks to } 6 \text { months }\end{array}$ & 915 & $\begin{array}{l}52.3 \text { - visual field loss } \\
54 \text { - complete } \\
\text { homonymous hemianopia } \\
19.5 \text { - partial } \\
\text { homonymous hemianopia } \\
15.2 \text {-homonymous } \\
\text { quadrantanopia } \\
0.2 \text { - temporal crescent } \\
9.2 \text { - constricted fields } \\
5.1 \text { - scotomas } \\
1.7 \text { - bilateral hemianopia }\end{array}$ & Yes & $\begin{array}{l}\text { Confrontation } \\
\text { Static perimetry } \\
\text { Kinetic perimetry }\end{array}$ \\
\hline $\begin{array}{l}\text { 2014; } \\
\text { Siong et } \\
\text { al. [61] }\end{array}$ & $\begin{array}{l}\text { Prospective } \\
\text { observation }\end{array}$ & General stroke & $\begin{array}{l}10 \text { days to } 26 \text { years } \\
\text { post stroke onset }\end{array}$ & 113 & $\begin{array}{l}26.5 \text { - monocular defects } \\
11.5 \text { - binocular defect }\end{array}$ & $\begin{array}{l}\text { Ocular } \\
\text { pathology }\end{array}$ & Confrontation \\
\hline $\begin{array}{l}2001 ; \\
\text { Lawrence } \\
\text { et al. [49] }\end{array}$ & Retrospective & Stroke register & $\begin{array}{l}\text { Median within } 3 \\
\text { months of onset }\end{array}$ & 1136 & 26.1 - visual field loss & Unknown & Unknown \\
\hline $\begin{array}{l}2002 ; \\
\text { Rathore et } \\
\text { al. [52] }\end{array}$ & Retrospective & $\begin{array}{l}\text { Database stroke } \\
\text { cohort }\end{array}$ & Unknown & 474 & $\begin{array}{l}14.6-\text { homonymous } \\
\text { hemianopia }\end{array}$ & Unknown & Unknown \\
\hline $\begin{array}{l}2005 ; \mathrm{Ng} \\
\text { et al. [50] }\end{array}$ & Retrospective & $\begin{array}{l}\text { Posterior } \\
\text { circulation strokes }\end{array}$ & Unknown & 89 & 53 - visual field loss & Unknown & Unknown \\
\hline $\begin{array}{l}\text { 2011; } \\
\text { Jerath } \\
\text { et al. [53] }\end{array}$ & Retrospective & $\begin{array}{l}\text { General stroke } \\
\text { Male vs female }\end{array}$ & Unknown & 449 & $\begin{array}{l}22.7-\text { visual field loss } \\
\text { (female) } \\
20.9-\text { visual field loss } \\
\text { (male) }\end{array}$ & Unknown & $\begin{array}{l}\text { Neurology } \\
\text { Accident \& } \\
\text { Emergency } \\
\text { assessment } \\
\text { Non-standardised }\end{array}$ \\
\hline $\begin{array}{l}\text { 2012; } \\
\text { Searls } \\
\text { et al. [42] }\end{array}$ & Retrospective & $\begin{array}{l}\text { Posterior } \\
\text { circulation stroke }\end{array}$ & Unknown & 407 & 22 - visual field loss & Unknown & $\begin{array}{l}\text { Neurology } \\
\text { assessment of } \\
\text { signs and } \\
\text { symptoms }\end{array}$ \\
\hline
\end{tabular}


Table 3. Eye movement disorder prevalence

\begin{tabular}{|c|c|c|c|c|c|c|c|}
\hline Study & Design & Population & $\begin{array}{l}\text { Time of vision } \\
\text { assessment }\end{array}$ & $\begin{array}{l}\text { Sample } \\
\text { size }(n=)\end{array}$ & $\begin{array}{l}\text { Prevalence of visual } \\
\text { issue (\%) }\end{array}$ & $\begin{array}{l}\text { Co-existent } \\
\text { ocular condition }\end{array}$ & $\begin{array}{l}\text { Method of } \\
\text { assessment }\end{array}$ \\
\hline $\begin{array}{l}\text { 1975; Yap } \\
\text { et al. [57] }\end{array}$ & $\begin{array}{l}\text { Prospective } \\
\text { observation }\end{array}$ & General stroke & $\begin{array}{l}\text { Median within } 2 \\
\text { days of onset }\end{array}$ & 100 & $\begin{array}{l}44 \text { - ocular motility } \\
\text { disorders } \\
28 \text { - gaze palsy } \\
11 \text { - impaired VOR } \\
6 \text { - cranial nerve palsy }\end{array}$ & Unknown & Unknown \\
\hline $\begin{array}{l}\text { 1982; De } \\
\text { Renzi } \\
\text { et al. [62] }\end{array}$ & $\begin{array}{l}\text { Prospective } \\
\text { observation }\end{array}$ & General stroke & $\begin{array}{l}\text { Follow-up every } 3- \\
4 \text { days for } 2 \text { weeks } \\
\text { post onset }\end{array}$ & 91 & $\begin{array}{l}28-\text { horizontal gaze } \\
\text { palsy } \\
7 \text { - nystagmus }\end{array}$ & Unknown & NIHSS \\
\hline $\begin{array}{l}\text { 1987; } \\
\text { Freeman \& } \\
\text { Rudge [35] }\end{array}$ & $\begin{array}{l}\text { Prospective } \\
\text { observation }\end{array}$ & General stroke & $\begin{array}{l}\text { Median within } 1 \\
\text { week of onset }\end{array}$ & 247 & $\begin{array}{l}22 \text { - ocular motility } \\
\text { disorders } \\
35 \text { - strabismus } \\
\text { (additional } 6 \% \text { pre- } \\
\text { existent) } \\
18 \text { - palsies } \\
\text { (skew deviation:3 } \\
11 / 2 \text { syndrome } 6 \\
\text { Horizontal gaze palsy } \\
57 \% \\
\text { Vertical gaze palsy } 20 \%] \\
23 \text { - nystagmus }\end{array}$ & Yes & $\begin{array}{l}\text { Medical } \\
\text { Orthoptic }\end{array}$ \\
\hline $\begin{array}{l}\text { 1995; Clisby } \\
\text { [32] }\end{array}$ & $\begin{array}{l}\text { Prospective } \\
\text { observation }\end{array}$ & General stroke & $\begin{array}{l}\text { Acute period on } \\
\text { stroke unit }\end{array}$ & 140 & $\begin{array}{l}52 \text { - strabismus } \\
44 \text { - gaze palsy: } \\
90 \text { - horizontal with right } \\
\text { hemisphere stroke } \\
73 \text { - horizontal with left } \\
\text { hemisphere stroke } \\
39 \text { - cranial nerve palsy } \\
\text { (mainly III) } \\
55-\text { reduced vergence } \\
\text { and stereoacuity }\end{array}$ & Ocular pathology & Orthoptic \\
\hline $\begin{array}{l}\text { 1996; Fowler } \\
\text { et al. [58] }\end{array}$ & $\begin{array}{l}\text { Prospective } \\
\text { observation }\end{array}$ & $\begin{array}{l}\text { Mixed } \\
\text { neurological on }\end{array}$ & $\begin{array}{l}\text { Median within } 2 \\
\text { months of }\end{array}$ & $\begin{array}{l}239(54 \% \\
\text { stroke ) }\end{array}$ & $\begin{array}{l}26-\text { stroke-related } \\
\text { strabismus }\end{array}$ & Unknown & Orthoptic \\
\hline
\end{tabular}


Hepworth et al.; OR, 5(1): 1-43, 2016; Article no.OR.21767

\begin{tabular}{|c|c|c|c|c|c|c|c|}
\hline Study & Design & Population & $\begin{array}{l}\text { Time of vision } \\
\text { assessment }\end{array}$ & $\begin{array}{l}\text { Sample } \\
\text { size }(n=)\end{array}$ & $\begin{array}{l}\text { Prevalence of visual } \\
\text { issue (\%) }\end{array}$ & $\begin{array}{l}\text { Co-existent } \\
\text { ocular condition }\end{array}$ & $\begin{array}{l}\text { Method of } \\
\text { assessment }\end{array}$ \\
\hline & & rehabilitation unit & admission & & & & \\
\hline $\begin{array}{l}\text { 2000; Lotery } \\
\text { et al. [64] }\end{array}$ & $\begin{array}{l}\text { Prospective } \\
\text { observation }\end{array}$ & General stroke & $\begin{array}{l}\text { Median within } 2 \\
\text { weeks of onset }\end{array}$ & 77 & 2.6 - third nerve palsy & Yes & $\begin{array}{l}\text { Ophthalmology } \\
\text { and optometric }\end{array}$ \\
\hline $\begin{array}{l}\text { 2006; Singer } \\
\text { et al. [63] }\end{array}$ & Prospective & $\begin{array}{l}\text { Sub population } \\
\text { excluding } \\
\text { haemorrhagic } \\
\text { stroke and } \\
\text { posterior } \\
\text { circulation } \\
\text { ischaemia }\end{array}$ & $\begin{array}{l}\text { Within } 6 \text { hours of } \\
\text { onset }\end{array}$ & 116 & $\begin{array}{l}26.7 \text { - complete gaze } \\
\text { palsy } \\
0.6 \text { - partial gaze palsy }\end{array}$ & Unknown & NIHSS \\
\hline $\begin{array}{l}\text { 2007; Rowe } \\
\text { et al. [70] }\end{array}$ & $\begin{array}{l}\text { Prospective } \\
\text { observation }\end{array}$ & $\begin{array}{l}\text { Stroke survivors } \\
\text { with suspected } \\
\text { visual } \\
\text { impairment }\end{array}$ & $\begin{array}{l}\text { Median within } 3 \\
\text { weeks of onset }\end{array}$ & 243 & $\begin{array}{l}54-\text { reduced } \\
\text { convergence }<6 \mathrm{cms} \text {. } \\
26-\text { reduced } \\
\text { convergence }<10 \mathrm{cms} \text {. }\end{array}$ & Yes & Orthoptic \\
\hline $\begin{array}{l}\text { 2008; Rowe } \\
\text { et al. [66] }\end{array}$ & $\begin{array}{l}\text { Prospective } \\
\text { observation }\end{array}$ & $\begin{array}{l}\text { Stroke survivors } \\
\text { with suspected } \\
\text { visual } \\
\text { impairment }\end{array}$ & $\begin{array}{l}\text { Median within } 3 \\
\text { weeks of onset }\end{array}$ & 323 & $\begin{array}{l}12-\text { nystagmus } \\
\mathrm{N}=2-\text { pre-existent } \\
\mathrm{N}=18 \text { - oscillopsia/vertigo } \\
\text { symptoms }\end{array}$ & Yes & Orthoptic \\
\hline $\begin{array}{l}2009 ; \\
\text { Siddique et } \\
\text { al. [65] }\end{array}$ & Prospective & General stroke & Acute period & 100 & $4-$ nystagmus & Unknown & $\begin{array}{l}\text { Unspecified } \\
\text { protocol }\end{array}$ \\
\hline $\begin{array}{l}\text { 2009; Akhtar } \\
\text { et al. [68] }\end{array}$ & Prospective & $\begin{array}{l}\text { Posterior } \\
\text { circulation stroke } \\
\text { only }\end{array}$ & Acute period & 116 & 48 - nystagmus & Unknown & Unknown \\
\hline $\begin{array}{l}\text { 2009; Rowe } \\
\text { et al. [24] }\end{array}$ & $\begin{array}{l}\text { Prospective } \\
\text { observation }\end{array}$ & $\begin{array}{l}\text { Stroke survivors } \\
\text { with suspected } \\
\text { visual } \\
\text { impairment }\end{array}$ & $\begin{array}{l}\text { Median within } 3 \\
\text { weeks of onset }\end{array}$ & 323 & $\begin{array}{l}54-\text { reduced } \\
\text { convergence }<6 \mathrm{cms} \\
26-\text { reduced } \\
\text { convergence }<10 \mathrm{cms}\end{array}$ & Yes & Orthoptic \\
\hline $\begin{array}{l}\text { 2010; Rowe } \\
\text { et al. [23] }\end{array}$ & $\begin{array}{l}\text { Prospective } \\
\text { observation }\end{array}$ & $\begin{array}{l}\text { Stroke survivors } \\
\text { with suspected } \\
\text { visual } \\
\text { impairment }\end{array}$ & $\begin{array}{l}\text { Median within } 3 \\
\text { weeks of onset }\end{array}$ & 512 & $\begin{array}{l}19 \text { - strabismus } \\
16.5-\text { new onset } \\
2.5 \text { - pre-existent }\end{array}$ & Yes & Orthoptic \\
\hline $\begin{array}{l}\text { 2011; Rowe } \\
\text { et al. }\end{array}$ & $\begin{array}{l}\text { Prospective } \\
\text { observation }\end{array}$ & $\begin{array}{l}\text { Stroke survivors } \\
\text { with suspected }\end{array}$ & $\begin{array}{l}\text { Median within } 3 \\
\text { weeks of onset }\end{array}$ & 915 & $\begin{array}{l}54 \text { - ocular motility } \\
\text { disorders }\end{array}$ & Yes & Orthoptic \\
\hline
\end{tabular}


Hepworth et al.; OR, 5(1): 1-43, 2016; Article no.OR.21767

\begin{tabular}{|c|c|c|c|c|c|c|c|}
\hline Study & Design & Population & $\begin{array}{l}\text { Time of vision } \\
\text { assessment }\end{array}$ & $\begin{array}{l}\text { Sample } \\
\text { size }(n=)\end{array}$ & $\begin{array}{l}\text { Prevalence of visual } \\
\text { issue (\%) }\end{array}$ & $\begin{array}{l}\text { Co-existent } \\
\text { ocular condition }\end{array}$ & $\begin{array}{l}\text { Method of } \\
\text { assessment }\end{array}$ \\
\hline$[18,19]$ & & $\begin{array}{l}\text { visual } \\
\text { impairment }\end{array}$ & & & $\begin{array}{l}2 / 3 \text { - diplopia } \\
19-\text { strabismus }(2.5 \% \\
\text { pre-existent) } \\
10-\text { cranial nerve palsy } \\
(\mathrm{VI}>\mathrm{III}>\mathrm{IV}) \\
58-\mathrm{VI} \\
26-\mathrm{III}\end{array}$ & & \\
\hline $\begin{array}{l}\text { 2011; Baier } \\
\text { \& Dieterich } \\
\text { [67] }\end{array}$ & Prospective & Cerebellar stroke & Mean within 6 days & 21 & $33-$ nystagmus & Unknown & $\begin{array}{l}\text { Eye movement } \\
\text { recording }\end{array}$ \\
\hline $\begin{array}{l}\text { Maeshima et } \\
\text { al. [59] }\end{array}$ & $\begin{array}{l}\text { Prospective } \\
\text { observation }\end{array}$ & Pontine stroke & Unknown & 68 & 15.9 - diplopia & Unknown & Unknown \\
\hline $\begin{array}{l}\text { 2012; Tao et } \\
\text { al. [43] }\end{array}$ & $\begin{array}{l}\text { Prospective } \\
\text { observation }\end{array}$ & $\begin{array}{l}\text { General stroke: } \\
\text { Anterior vs } \\
\text { posterior } \\
\text { circulation stroke }\end{array}$ & Acute period & 1174 & $\begin{array}{l}\text { 8- diplopia: } \\
\text { 7.3 posterior circulation } \\
0.7 \text { anterior circulation } \\
13.5 \text { - gaze palsy: } \\
11 \text { - anterior circulation } \\
2.6 \text { - posterior circulation } \\
4-\text { cranial nerve palsy: } \\
\text { posterior circulation }\end{array}$ & Unknown & NIHSS \\
\hline $\begin{array}{l}2013 ; \text { Su \& } \\
\text { Young [60] }\end{array}$ & $\begin{array}{l}\text { Prospective } \\
\text { observation }\end{array}$ & $\begin{array}{l}\text { Posterior fossa } \\
\text { stroke: vertigo } \\
\text { clinic }\end{array}$ & Unknown & 70 & $\begin{array}{l}31 \text { - ocular motility } \\
\text { disorders } \\
45-\text { diplopia } \\
\mathrm{N}=22 \text { - nystagmus } \\
{[45.5 \% \text { multidirectional }} \\
54.5 \text { unidirectional } \\
86 \text { - reduced OKN] }\end{array}$ & Unknown & $\begin{array}{l}\text { Nystagmus - eye } \\
\text { movement } \\
\text { recordings }\end{array}$ \\
\hline $\begin{array}{l}\text { 2013; Rowe } \\
\text { et al. [22] }\end{array}$ & $\begin{array}{l}\text { Prospective } \\
\text { observation }\end{array}$ & $\begin{array}{l}\text { Stroke survivors } \\
\text { with suspected } \\
\text { visual } \\
\text { impairment }\end{array}$ & $\begin{array}{l}\text { Median within } 3 \\
\text { weeks of onset }\end{array}$ & 915 & $\begin{array}{l}23 \text { - gaze defect: } \\
15.9 \text { - horizontal and } \\
\text { vertical gaze palsy } \\
69.7 \text { - complete } \\
13.5 \text { - saccadic palsy } \\
22.2 \text { - smooth pursuit }\end{array}$ & Yes & Orthoptic \\
\hline
\end{tabular}


Hepworth et al.; OR, 5(1): 1-43, 2016; Article no.OR.21767

\begin{tabular}{|c|c|c|c|c|c|c|c|}
\hline Study & Design & Population & $\begin{array}{l}\text { Time of vision } \\
\text { assessment }\end{array}$ & $\begin{array}{l}\text { Sample } \\
\text { size }(n=)\end{array}$ & $\begin{array}{l}\text { Prevalence of visual } \\
\text { issue (\%) }\end{array}$ & $\begin{array}{l}\text { Co-existent } \\
\text { ocular condition }\end{array}$ & $\begin{array}{l}\text { Method of } \\
\text { assessment }\end{array}$ \\
\hline & & & & & $\begin{array}{l}\text { palsy } \\
22.2 \text { - impaired gaze } \\
\text { holding } \\
3.9 \text { - Parinaud's } \\
\text { syndrome } \\
9.7 \text { - INO } \\
1.4 \text { - one and a half } \\
\text { syndrome }\end{array}$ & & \\
\hline $\begin{array}{l}\text { 2014; Siong } \\
\text { et al. [61] }\end{array}$ & $\begin{array}{l}\text { Prospective } \\
\text { observation }\end{array}$ & General stroke & $\begin{array}{l}10 \text { days to } 26 \\
\text { years post stroke } \\
\text { onset }\end{array}$ & 113 & $\begin{array}{l}53.1 \text { - jerky eye } \\
\text { movements } \\
11.5-\text { restricted ocular } \\
\text { motility } \\
20-\text { reduced } \\
\text { convergence }(<15 \mathrm{~cm})\end{array}$ & Yes & Optometrist \\
\hline $\begin{array}{l}2011 \text {; Jerath } \\
\text { et [53] }\end{array}$ & Retrospective & $\begin{array}{l}\text { General stroke } \\
\text { Male vs female }\end{array}$ & Unknown & 449 & $\begin{array}{l}7.8 \text { - diplopia }(7.1 \% \\
\text { male, } 0.7 \% \text { female }) \\
17.5-\text { nystagmus } \\
(4.6 \text { male, } 12.9 \text { female })\end{array}$ & Unknown & $\begin{array}{l}\text { Neurology } \\
\text { Accident \& } \\
\text { Emergency } \\
\text { assessment } \\
\text { Non- } \\
\text { standardised }\end{array}$ \\
\hline $\begin{array}{l}\text { 2012; Searls } \\
\text { et al. [42] }\end{array}$ & Retrospective & $\begin{array}{l}\text { Posterior } \\
\text { circulation stroke }\end{array}$ & Unknown & 407 & $\begin{array}{l}20 \text { - ocular motility } \\
\text { disorders } \\
15 \text { - diplopia } \\
25 \text { - nystagmus }\end{array}$ & Unknown & $\begin{array}{l}\text { Neurology } \\
\text { assessment of } \\
\text { signs and } \\
\text { symptoms }\end{array}$ \\
\hline
\end{tabular}


Table 4. Central visual deficit prevalence

\begin{tabular}{|c|c|c|c|c|c|c|c|}
\hline Study & Design & Population & $\begin{array}{l}\text { Time of vision } \\
\text { assessment }\end{array}$ & $\begin{array}{l}\text { Sample } \\
\text { size }(n=)\end{array}$ & $\begin{array}{l}\text { Prevalence of visual } \\
\text { issue (\%) }\end{array}$ & $\begin{array}{l}\text { Co-existent } \\
\text { ocular } \\
\text { condition }\end{array}$ & $\begin{array}{l}\text { Method of } \\
\text { assessment }\end{array}$ \\
\hline $\begin{array}{l}1989 ; \\
\text { Bulens et } \\
\text { al. [71] }\end{array}$ & $\begin{array}{l}\text { Prospective } \\
\text { observation }\end{array}$ & General stroke & $\begin{array}{l}\text { Days to years post } \\
\text { onset }\end{array}$ & 16 & $\begin{array}{l}62-\text { reduced contrast } \\
\text { sensitivity }\end{array}$ & No & Ophthalmology \\
\hline $\begin{array}{l}\text { 1995; } \\
\text { Clisby [32] }\end{array}$ & $\begin{array}{l}\text { Prospective } \\
\text { observation }\end{array}$ & General stroke & $\begin{array}{l}\text { Acute period on } \\
\text { stroke unit }\end{array}$ & 140 & $\begin{array}{l}58 \text { - reduced visual } \\
\text { acuity }\end{array}$ & $\begin{array}{l}\text { Excluded } \\
\text { ocular } \\
\text { pathology }\end{array}$ & $\begin{array}{l}\text { Orthoptic with } \\
\text { adapted visual acuity } \\
\text { assessment for } \\
\text { dysphasia }\end{array}$ \\
\hline $\begin{array}{l}\text { 2000; } \\
\text { Lotery } \\
\text { et al. [64] }\end{array}$ & $\begin{array}{l}\text { Prospective } \\
\text { observation }\end{array}$ & General stroke & $\begin{array}{l}\text { Median within } 2 \\
\text { weeks of onset }\end{array}$ & 77 & $\begin{array}{l}30 \text { - visual acuity } \leq 6 / 12 \\
27 \text { - no glasses } \\
\text { available, dirty or } \\
\text { damaged lenses }\end{array}$ & Yes & $\begin{array}{l}\text { Ophthalmology and } \\
\text { optometric }\end{array}$ \\
\hline $\begin{array}{l}\text { 2006; } \\
\text { Edwards et } \\
\text { al. [70] }\end{array}$ & $\begin{array}{l}\text { Prospective } \\
\text { observation }\end{array}$ & $\begin{array}{l}\text { General stroke with } \\
\text { exclusions if } \\
\text { unable to hold a } \\
\text { pencil or severe } \\
\text { motor or language } \\
\text { deficits }\end{array}$ & $\begin{array}{l}\text { Median within } 15 \\
\text { days of onset }\end{array}$ & 53 & $\begin{array}{l}70-\text { reduced visual } \\
\text { acuity } \\
30-6 / 7.5-6 / 15 \\
4-6 / 21-6 / 30 \\
36-6 / 60-6 / 120 \\
54-\text { no glasses } \\
\text { available }\end{array}$ & Unknown & Near visual acuity \\
\hline $\begin{array}{l}\text { 2011; Rowe } \\
\text { et al. [19] }\end{array}$ & $\begin{array}{l}\text { Prospective } \\
\text { observation }\end{array}$ & $\begin{array}{l}\text { Stroke survivors } \\
\text { with suspected } \\
\text { visual impairment }\end{array}$ & $\begin{array}{l}\text { Median within } 3 \\
\text { weeks of onset }\end{array}$ & 915 & $\begin{array}{l}19.3 \text { - reading } \\
\text { impairment: } \\
61.6 \text { - field loss } \\
45.8 \text { - reduced } \\
\text { convergence } \\
45 \text { - saccadic defects } \\
22.5 \text { - reduced visual } \\
\text { acuity } \\
22 \text { - perceptual defect }\end{array}$ & Yes & Orthoptic \\
\hline $\begin{array}{l}2013 a ; \\
\text { Rowe et al. } \\
{[36]}\end{array}$ & $\begin{array}{l}\text { Prospective } \\
\text { observation }\end{array}$ & $\begin{array}{l}\text { Stroke survivors } \\
\text { with suspected } \\
\text { visual impairment }\end{array}$ & $\begin{array}{l}\text { Median within } 3 \\
\text { weeks of onset }\end{array}$ & 915 & $\begin{array}{l}31-\text { reduced visual } \\
\text { acuity }\end{array}$ & Yes & Orthoptic \\
\hline
\end{tabular}


Hepworth et al.; OR, 5(1): 1-43, 2016; Article no.OR.21767

\begin{tabular}{|c|c|c|c|c|c|c|c|}
\hline Study & Design & Population & $\begin{array}{l}\text { Time of vision } \\
\text { assessment }\end{array}$ & $\begin{array}{l}\text { Sample } \\
\text { size }(n=)\end{array}$ & $\begin{array}{l}\text { Prevalence of visual } \\
\text { issue (\%) }\end{array}$ & $\begin{array}{l}\text { Co-existent } \\
\text { ocular } \\
\text { condition }\end{array}$ & $\begin{array}{l}\text { Method of } \\
\text { assessment }\end{array}$ \\
\hline $\begin{array}{l}\text { 2011; } \\
\text { Jerath et al. } \\
{[53]}\end{array}$ & Retrospective & $\begin{array}{l}\text { General stroke } \\
\text { Male vs female }\end{array}$ & Unknown & 449 & $\begin{array}{l}27 \text { - loss of vision } \\
\text { reported: } \\
15.8 \text { - male } \\
10.3 \text { - female } \\
19 \text { - visual disturbance } \\
\text { reported: blurred vision, } \\
\text { focus difficulty, } \\
\text { photophobia, visual } \\
\text { hallucinations }\end{array}$ & Unknown & $\begin{array}{l}\text { Neurology } \\
\text { Accident \& } \\
\text { Emergency } \\
\text { assessment } \\
\text { Non-standardised }\end{array}$ \\
\hline $\begin{array}{l}\text { 2012; } \\
\text { Searls et al. } \\
{[42]}\end{array}$ & Retrospective & $\begin{array}{l}\text { Posterior } \\
\text { circulation stroke }\end{array}$ & Unknown & 407 & 20 - blurred vision & Unknown & $\begin{array}{l}\text { Neurology } \\
\text { assessment of signs } \\
\text { and symptoms }\end{array}$ \\
\hline $\begin{array}{l}\text { 2012; dos } \\
\text { Santos \& } \\
\text { Andrade } \\
{[72]}\end{array}$ & Retrospective & $\begin{array}{l}\text { General stroke with } \\
\text { haemorrhagic } \\
\text { stroke excluded }\end{array}$ & & 40 & $\begin{array}{l}100-\text { reduced contrast } \\
\text { in comparison to } \\
\text { controls }\end{array}$ & $\begin{array}{l}\text { Excluded } \\
\text { ocular } \\
\text { pathology }\end{array}$ & Ophthalmology \\
\hline $\begin{array}{l}\text { 2014; Siong } \\
\text { et al. [61] }\end{array}$ & $\begin{array}{l}\text { Prospective } \\
\text { observation }\end{array}$ & General stroke & $\begin{array}{l}10 \text { days to } 26 \\
\text { years post stroke } \\
\text { onset }\end{array}$ & 113 & $\begin{array}{l}29.8-\text { vision worse } \\
\text { than } 0.3 \text { LogMAR } \\
11.5-\text { mild reduced } \\
\text { vision (worse than } 0.5 \\
\text { LogMAR) } \\
1.8 \text { - moderate } \\
\text { reduced vision (worse } \\
\text { than } 1.0 \text { LogMAR) }\end{array}$ & Yes & Optometrist \\
\hline
\end{tabular}


Visual inattention has been reported on average in $32 \%$ (range $14 \%$ to $82 \%$ ) (Table 5 ) of stroke survivors from five studies $(n=1800)$ [56,73-76]. These studies have recruited participants consecutively and have used a range of tests or tools for visual inattention including cancellation tests and the Behavioural Inattention Test. Studies $(n=1335)$ using cancellation tests alone reported prevalence of $15 \%$ to $26 \%[73,75,77]$. Those using a variety of assessments ( $n=991)$ for visual inattention reported a prevalence of $14 \%$ to $82 \%$ [56,74,78-81]. Discrepancies in the wide range of prevalence figures typically related to the timing of assessment plus inclusion/exclusion criteria of left versus right sided stroke lesions and severe cognitive and/or communication deficits. As expected, there was a greater prevalence of left versus right sided inattention.

In addition to visual neglect/inattention, the prevalence of other perceptual deficits are reported in the literature. Perceptual deficits, such as object agnosia, colour detection difficulties have been reported in the literature in very small numbers $[19,23,24,81]$. Our literature search found four studies reporting an estimated prevalence for different visual perceptual deficits following stroke [24]. Beaudoin et al. $(n=189)$ reported an overall prevalence of visual perception deficits as $49.2 \%$ [82]. Rowe et al. $(n=323)$ estimated the prevalence as $20 \%$, of which the prevalence of visual hallucinations after stroke was $4 \%$ and visual agnosia was $2.5 \%$ [24]. It was reported that patients with visual hallucinations and other perceptual deficits frequently do not disclose these symptoms. This, in addition to the method of recruitment could result in an under-estimation of the true prevalence. Yang et al. ( $n=82)$ reported $50 \%$ of participants had pathologic ( $\left.>3^{\circ}\right)$ subjective visual vertical tilt following brainstem stroke [83]. Chechlacz et al. ( $n=454)$ reported $28 \%$ of participants with right hemisphere stroke showed left visual extinction versus $6.8 \%$ of participants with left hemisphere stroke showed right visual extinction [84].

Freeman and Rudge reported $79 \%$ of participants to have defective stereopsis [35]. Stereopsis was only tested in the pilot study $(n=26)$, therefore the number of participants tested was limited to 19. It was also purposely not tested on participants with manifest strabismus even those which were a direct result of the stroke. The majority of those with strabismus would not demonstrate any stereopsis. This would result in an underestimation of those suffering reduced or absent stereopsis as a direct result of stroke.

\section{RECOVERY OF VISUAL FUNCTION}

Our literature search identified just one study that appears to report the recovery of overall visual problems following stroke (Table 6). The majority that report recovery do so for visual field loss (Table 7). Ali et al. had the largest sample for tracking recovery of multiple visual problems following stroke [30]. However, not all visual problems were included due to the use of the NIHSS which limits assessment to visual field loss and horizontal gaze paresis. There was a variable sample size at the three time points used (baseline, 30 days and 90 days post stroke). The authors reported a reduction of visual problems to $28.2 \%$ at 30 days and a further reduction to $20.5 \%$ at 90 days, compared to the initial $60.5 \%$ at baseline. The sample size considerably decreased between baseline $(n=11,900)$ to 30 days post stroke $(n=4,965)$.

\subsection{Visual Field Loss}

Recovery of visual field loss is reported by a number of studies but across variable time periods (Table 7). The percentage of patients recovering from visual field loss ranges from $0 \%$ to $44 \%$ for complete recovery and up to $72.2 \%$ for partial recovery $(n=6656)[30,35,41,46,55$, 85-87]. Variability in recovery rates appears to be dependent on time of baseline assessment and length of follow-up, accuracy of visual field assessment methods and their sensitivity to detection of change, prospective versus retrospective studies and exclusions of severe neurological and communication defects.

Gray et al. $(n=174)$ documented recovery in $47.8 \%$ of their sample, with a slightly higher proportion of $56.5 \%$ who had suffered a right hemianopia [41]. The macula was involved in $56.3 \%$ of the sample; $72.2 \%$ seeing an improvement in this and surrounding areas. They noted four different patterns of recovery, the most common $(34.4 \%)$ of which was recovery of the lower quadrant. This was followed by complete recovery $(25 \%)$, recovery of the upper quadrant $(21.9 \%)$ and finally improvement in both quadrants with some residual defect (18.7\%). They found that most improvement occurred between 6 and 25 days post stroke. Cassidy et al. $(n=19)$ reported that of those patients who demonstrated some recovery, only $15.8 \%$ 
achieved complete recovery at four weeks [46]. The majority of $42.1 \%$ had some central recovery and the remainder had quadrantic recovery. For a patient with complete homonymous hemianopia the recovery of the macula area can appear to be only a small recovery. However, this can have a considerable functional impact such as with reading ability. They were also able to demonstrate the reduced sensitivity of the confrontation method at detecting areas of recovery. Variances in reports related to whether the baseline visual field loss was complete or partial and/or congruous versus incongruous loss along with stroke-specific or mixed populations.

\subsection{Ocular Motility Abnormalities and Strabismus}

Less has been reported on the recovery of ocular alignment and motility problems following a stroke (Table 8). The percentage of patients which were reported to recover ranged from $7 \%$ to $28.5 \%$ for full recovery and up to $92 \%$ for partial recovery $(n=6047) \quad[18,22,30,35,62,66]$. The greatest recovery was for reduced stereoacuity at $92 \%$ [35]. Sixth nerve palsies were reported to have the highest incidence of complete recovery of cranial nerve palsies at $28.5 \%$ [18]. At least one third showed no recovery across ocular motility conditions of gaze palsy, nystagmus, cranial nerve palsy and strabismus $[18,19,35,66]$.

\subsection{Visual Acuity and Central Vision Deficit}

Little is reported on the recovery of vision following stroke (Table 9). We found one study $(n=247)$ that outlined the recovery of reduced vision following stroke [35]. The majority (71\%) showed some recovery. It is not clear from this paper what extent of recovery was made and whether this had been achieved at the one or six month follow-up.

Rowe et al. $(n=915)$ reported the recovery rates for a group of participants suffering reading difficulties [19]. The data from follow-up visits was available for $42.9 \%$ of the participants. Of these, $10.5 \%$ had complete resolution of their symptoms, and $43.4 \%$ showed some improvement. A similar proportion of $44.7 \%$ saw no change in their symptoms and only $1.3 \%$ experienced deterioration in their condition.

\subsection{Visual Perception abnormalities}

\subsubsection{Visual inattention}

Four studies $(n=5286)$ have reported recovery of visual neglect/inattention $[30,35,79,88]$. The percentage of recovery reported in the literature ranges from $29 \%$ to $78 \%$ (Table 10). In contrast to other visual impairments, patients suffering with visual neglect were more likely to require a longer stay in hospital and have a poorer prognosis for recovering function [73]. Recovery is mostly seen within 3 months post onset $[30,35,79]$ with approximately $10 \%$ full recovery within the first 2 weeks [90].

\subsubsection{Other perceptual deficits}

One study $(n=140)$ was found to report the recovery of visual hallucinations [89]. The authors reported that visual hallucinations (Charles Bonnet syndrome) persisted for several days or weeks after the onset of stroke before gradually subsiding. The median duration of visual hallucinations was 28 days and they stated that the first 90 days is when spontaneous recovery is most likely to occur.

\section{LIMITATIONS AND RECOMMENDA- TIONS FOR FUTURE INCIDENCE, PREVALENCE AND RECOVERY STUDIES}

None of the studies provided information about stroke survivors who were not admitted to a stroke unit/ward/rehabilitation unit. It is acknowledged that a proportion of stroke survivors have visual impairment only (usually occipital infarcts) but the numbers of these remain unknown.

The time of visual examination post stroke has a direct effect on the estimate of prevalence of visual problems that occur due to stroke. As recovery of visual conditions can occur rapidly in some cases during the first weeks post stroke, studies that assess visual function later than this early two week period are likely to detect those with persistent visual impairment. The extent of visual impairment for those with persistent visual conditions may also be misrepresented as these individuals may have had substantial improvement with only partial deficits remaining. Thus there is considerable potential for an underestimation of stroke related visual impairment. 
Table 5. Visual perceptual impairment prevalence

\begin{tabular}{|c|c|c|c|c|c|c|c|}
\hline Study & Design & Population & $\begin{array}{l}\text { Time of vision } \\
\text { assessment }\end{array}$ & $\begin{array}{l}\text { Sample } \\
\text { size }(n=)\end{array}$ & $\begin{array}{l}\text { Prevalence of } \\
\text { visual issue (\%) }\end{array}$ & $\begin{array}{l}\text { Co-existent } \\
\text { ocular } \\
\text { condition }\end{array}$ & $\begin{array}{l}\text { Method of } \\
\text { assessment }\end{array}$ \\
\hline $\begin{array}{l}\text { 1987; Freeman } \\
\text { \& Rudge [35] }\end{array}$ & $\begin{array}{l}\text { Prospective } \\
\text { observation }\end{array}$ & General stroke & $\begin{array}{l}\text { Median within } 1 \\
\text { week of onset }\end{array}$ & 247 & $\begin{array}{l}79-\text { reduced } \\
\text { stereoacuity }\end{array}$ & Yes & Orthoptic \\
\hline $\begin{array}{l}1993 ; \text { Stone et } \\
\text { al. [56] }\end{array}$ & Prospective & General stroke & $\begin{array}{l}\text { Median within } 3 \\
\text { days of onset }\end{array}$ & 171 & $\begin{array}{l}82-\text { visual neglect } \\
\text { [right hemisphere] } \\
65-\text { visual neglect } \\
\text { [left hemisphere] } \\
28-\text { anosognosia } \\
\text { [right hemisphere] } \\
5-\text { anosognosia [left } \\
\text { hemisphere] }\end{array}$ & Unknown & $\begin{array}{l}\text { Modified } \\
\text { behavioural } \\
\text { inattention test }\end{array}$ \\
\hline $\begin{array}{l}\text { 1997; Pedersen } \\
\text { et al. [73] }\end{array}$ & Prospective & General stroke & At admission & 1014 & $\begin{array}{l}23-\text { visual neglect } \\
\text { [42 - right } \\
\text { hemisphere, } 8 \text { - left } \\
\text { hemisphere] }\end{array}$ & Unknown & Cancellation tasks \\
\hline $\begin{array}{l}\text { 1998; Cassidy } \\
\text { et al. [79] }\end{array}$ & Prospective & $\begin{array}{l}\text { General stroke } \\
\text { with left } \\
\text { hemisphere } \\
\text { lesions excluded }\end{array}$ & $\begin{array}{l}\text { Within } 7 \text { days and } \\
\text { monthly follow-up }\end{array}$ & 66 & $\begin{array}{l}40.9 \text { - visual neglect } \\
74-\text { visual field loss }\end{array}$ & Unknown & $\begin{array}{l}\text { Behavioural } \\
\text { inattention test }\end{array}$ \\
\hline $\begin{array}{l}\text { 1999; Cassidy } \\
\text { et al. [80] }\end{array}$ & Prospective & $\begin{array}{l}\text { General stroke } \\
\text { with left } \\
\text { hemisphere } \\
\text { lesions excluded }\end{array}$ & $\begin{array}{l}\text { Within } 7 \text { days and } \\
\text { monthly follow-up }\end{array}$ & 44 & $61.4-$ visual neglect & Unknown & $\begin{array}{l}\text { Behavioural } \\
\text { inattention test }\end{array}$ \\
\hline $\begin{array}{l}\text { 2002; Appleros } \\
\text { et al. [74] }\end{array}$ & $\begin{array}{l}\text { Prospective } \\
\text { retrospective } \\
\text { cases }\end{array}$ & General stroke & Unknown & 279 & $\begin{array}{l}23-\text { visual neglect } \\
\text { [62 - right } \\
\text { hemisphere] } \\
74 \text { - anosognosia }\end{array}$ & Unknown & Test battery \\
\hline $\begin{array}{l}\text { 2006; Linden et } \\
\text { al. [75] }\end{array}$ & Prospective & General stroke & $\begin{array}{l}\text { At } 20 \text { months of } \\
\text { onset }\end{array}$ & 243 & $15-$ visual neglect & Unknown & Star cancellation \\
\hline $\begin{array}{l}\text { 2007; Becker \& } \\
\text { Karnath [76] }\end{array}$ & Prospective & General stroke & $\begin{array}{l}\text { Median within } 3 \\
\text { days of onset }\end{array}$ & 93 & $\begin{array}{l}26.2-\text { visual neglect } \\
\text { [right hemisphere] } \\
24.3-\text { visual } \\
\text { extinction }\end{array}$ & Unknown & Cancellation tasks \\
\hline
\end{tabular}


Hepworth et al.; OR, 5(1): 1-43, 2016; Article no.OR.21767

\begin{tabular}{|c|c|c|c|c|c|c|c|}
\hline Study & Design & Population & $\begin{array}{l}\text { Time of vision } \\
\text { assessment }\end{array}$ & $\begin{array}{l}\text { Sample } \\
\text { size }(n=)\end{array}$ & $\begin{array}{l}\text { Prevalence of } \\
\text { visual issue (\%) }\end{array}$ & $\begin{array}{l}\text { Co-existent } \\
\text { ocular } \\
\text { condition }\end{array}$ & $\begin{array}{l}\text { Method of } \\
\text { assessment }\end{array}$ \\
\hline & & & & & $\begin{array}{l}2.4-\text { visual neglect } \\
\text { [left hemisphere] } \\
4.9-\text { visual } \\
\text { extinction }\end{array}$ & & \\
\hline $\begin{array}{l}\text { 2009; Lee et al. } \\
\text { [78] }\end{array}$ & Prospective & $\begin{array}{l}\text { General stroke } \\
\text { Left hemisphere } \\
\text { excluded }\end{array}$ & $\begin{array}{l}\text { Median within } 2 \\
\text { months of onset }\end{array}$ & 138 & $\begin{array}{l}58-\text { visual neglect } \\
22.5 \text { - neglect } \\
\text { dyslexia }\end{array}$ & Unknown & Test battery \\
\hline $\begin{array}{l}\text { 2009; van Nes } \\
\text { et al. [77] }\end{array}$ & Prospective & $\begin{array}{l}\text { General stroke } \\
\text { Excluded } \\
\text { aphasia, gaze } \\
\text { palsy, cognitive } \\
\text { issues }\end{array}$ & $\begin{array}{l}\text { Median within } 2 \\
\text { weeks of onset }\end{array}$ & 78 & $\begin{array}{l}21.8 \text { - visual neglect } \\
88 \text { - right } \\
\text { hemisphere }\end{array}$ & $\begin{array}{l}\text { Gaze paresis } \\
\text { excluded }\end{array}$ & Cancellation tasks \\
\hline $\begin{array}{l}\text { 2009; Rowe et } \\
\text { al. }[6,24]\end{array}$ & Prospective & $\begin{array}{l}\text { Stroke survivors } \\
\text { with suspected } \\
\text { visual defect }\end{array}$ & $\begin{array}{l}\text { Median within } 3 \\
\text { weeks of onset }\end{array}$ & 323 & $\begin{array}{l}14-\text { visual neglect } \\
4-\text { visual } \\
\text { hallucinations } \\
2.5-\text { visual agnosia }\end{array}$ & Yes & Test battery \\
\hline $\begin{array}{l}\text { 2013; Beaudoin } \\
\text { et al. [82] }\end{array}$ & $\begin{array}{l}\text { Prospective } \\
\text { longitudinal }\end{array}$ & General stroke & $\begin{array}{l}\text { At discharge to } \\
\text { home }\end{array}$ & 189 & $\begin{array}{l}49.2 \text { - visual } \\
\text { perceptual defect }\end{array}$ & Unknown & $\begin{array}{l}\text { Motor-free visual } \\
\text { perceptual test- } \\
\text { vertical version }\end{array}$ \\
\hline $\begin{array}{l}2014 ; \\
\text { Chechlacz et al. } \\
{[84]}\end{array}$ & $\begin{array}{l}\text { Prospective } \\
\text { observational }\end{array}$ & Sub-acute stroke & $2.5-27.3$ days & 454 & $\begin{array}{l}9.1-\text { left visual } \\
\text { extinction } \\
4.6 \text { right visual } \\
\text { extinction }\end{array}$ & Unknown & $\begin{array}{l}\text { Confrontation } \\
\text { extinction }\end{array}$ \\
\hline $\begin{array}{l}\text { 2014; Siong et } \\
\text { al. [61] }\end{array}$ & $\begin{array}{l}\text { Prospective } \\
\text { observational }\end{array}$ & General stroke & $\begin{array}{l}10 \text { days to } 26 \text { years } \\
\text { post stroke onset }\end{array}$ & 113 & 5.3 visual neglect & Yes & Line bisection \\
\hline $\begin{array}{l}\text { 2014; Yang et } \\
\text { al. [83] }\end{array}$ & $\begin{array}{l}\text { Prospective } \\
\text { observational }\end{array}$ & $\begin{array}{l}\text { Brainstem } \\
\text { infarction }\end{array}$ & $\begin{array}{l}\text { Less than } 10 \text { days } \\
\text { post symptom } \\
\text { onset }\end{array}$ & 82 & $\begin{array}{l}50-\text { pathologic } \\
\text { subjective visual } \\
\text { vertical tilt }\left(>3^{\circ}\right) \\
76 \text { - ipsiversive } \\
24-\text { contraversive } \\
54.7 \text { - abnormal } \\
\text { torsion }\end{array}$ & Unknown & $\begin{array}{l}\text { Computerised } \\
\text { assessment }\end{array}$ \\
\hline
\end{tabular}




\section{Table 6. Recovery of visual impairment}

\begin{tabular}{|c|c|c|c|c|c|c|}
\hline Study & Design & Population & $\begin{array}{l}\text { Time of vision } \\
\text { assessment }\end{array}$ & Sample size $(n=)$ & Prevalence of visual issue (\%) & Assessment \\
\hline $\begin{array}{l}\text { 2013; Ali et al. } \\
\text { [30] }\end{array}$ & Prospective & $\begin{array}{l}\text { Stroke trial } \\
\text { database }\end{array}$ & $\begin{array}{l}\text { Baseline, } 30 \text { days and } \\
90 \text { days }\end{array}$ & $\begin{array}{l}11900 \text { at baseline } \\
4965 \text { at follow-up }\end{array}$ & $\begin{array}{l}28.2 \text { - visual impairment at } 30 \text { days } \\
20.5 \text { - visual impairment at } 90 \text { days } \\
\text { Versus } 60.6 \text { at baseline }\end{array}$ & NIHSS \\
\hline
\end{tabular}

Table 7. Recovery of visual field loss

\begin{tabular}{|c|c|c|c|c|c|c|}
\hline Study & Design & Population & Time of vision assessment & Sample size $(n=)$ & Prevalence of visual issue (\%) & Assessment \\
\hline $\begin{array}{l}\text { 1987; } \\
\text { Freeman } \\
\text { \& Rudge } \\
\text { [35] }\end{array}$ & Prospective & General stroke & $\begin{array}{l}\text { Mean } 73 \text { day follow-up } 1 \text { week to } 6 \\
\text { months }\end{array}$ & 247 & $\begin{array}{l}33 \text { - improvement ( } 22 \text { full, } 11 \\
\text { partial) } \\
25 \text { - stable field }\end{array}$ & Confrontation \\
\hline $\begin{array}{l}\text { 1989; } \\
\text { Gray et al. } \\
{[41]}\end{array}$ & Prospective & General stroke & $\begin{array}{l}\text { Followed every } 24 \text { hours for } 4 \text { days } \\
\text { and max to } 28 \text { days }\end{array}$ & 174 & $\begin{array}{l}\text { Complete hemianopia: } \\
17 \text { - full resolution within } 2-10 \text { days } \\
27 \text { - partial improvement } \\
39 \text { - stable field } \\
\text { Partial hemianopia: } \\
44 \text { - full resolution within } 48 \text { hours } \\
28 \text { - full resolution within } 14 \text { days } \\
17 \text { - stable field }\end{array}$ & Confrontation \\
\hline $\begin{array}{l}\text { 1991; Tiel } \\
\text { \& Kolmel } \\
{[85]}\end{array}$ & Prospective & $\begin{array}{l}\text { Posterior } \\
\text { circulation } \\
\text { stroke } \\
\text { Excluded } \\
\text { communication } \\
\text { difficulty and } \\
\text { severe } \\
\text { neurological } \\
\text { deficits }\end{array}$ & $\begin{array}{l}\text { Daily follow-up within } 3 \text { weeks of } \\
\text { onset }\end{array}$ & 125 & $\begin{array}{l}47.8 \text { - improvement within } 6-25 \\
\text { days } \\
56.5 \text { for right hemianopia } \\
56.3 \text { - macula involved with } 72.2 \\
\text { improvement of this } \\
34.4 \text { - recovery of lower quadrant } \\
25 \text { - full recovery } \\
21.9 \text { - recovery of upper quadrant } \\
18.7 \text { - partial recovery }\end{array}$ & Confrontation \\
\hline $\begin{array}{l}2001 ; \\
\text { Cassidy et }\end{array}$ & Prospective & General stroke & 4 week intervals up to 12 weeks & 19 & $\begin{array}{l}15.8 \text { - full recovery at } 4 \text { weeks } \\
42.1 \text { - central recovery }\end{array}$ & Perimetry \\
\hline
\end{tabular}


Hepworth et al.; OR, 5(1): 1-43, 2016; Article no.OR.21767

\begin{tabular}{|c|c|c|c|c|c|c|}
\hline Study & Design & Population & Time of vision assessment & Sample size $(n=)$ & Prevalence of visual issue (\%) & Assessment \\
\hline al. [46] & & & & & 11.1 - stable & \\
\hline $\begin{array}{l}\text { 2013; Ali } \\
\text { et al. [30] }\end{array}$ & Prospective & $\begin{array}{l}\text { Stroke trial } \\
\text { database }\end{array}$ & Baseline, 30 days and 90 days & $\begin{array}{l}11900 \text { at baseline } \\
4965 \text { at follow-up }\end{array}$ & $\begin{array}{l}\text { Complete hemianopia: } \\
13 \text { at } 30 \text { days } \\
10 \text { at } 90 \text { days } \\
\text { Versus } 35 \% \text { at baseline } \\
\text { Partial hemianopia: } \\
11 \text { at } 90 \text { days } \\
\text { Versus } 14.5 \% \text { at baseline }\end{array}$ & $\begin{array}{l}\text { NIHSS } \\
\text { Confrontation }\end{array}$ \\
\hline $\begin{array}{l}2006 \\
\text { Zhang et } \\
\text { al. [87] }\end{array}$ & Retrospective & $\begin{array}{l}\text { Mixed } \\
\text { population }\end{array}$ & $\begin{array}{l}\text { Median } 3 \text { months of onset } \\
\text { Change at } 3 \text { and } 6 \text { months }\end{array}$ & 254 & $\begin{array}{l}3 \text { - full recovery } \\
34-\text { partial } \\
63-\text { stable field }\end{array}$ & $\begin{array}{l}\text { Perimetry } \\
\text { Central } 30 \text { or } \\
24 \text { degrees } \\
\end{array}$ \\
\hline $\begin{array}{l}2007 ; \\
\text { Schmielau } \\
\& \text { Wong } \\
{[86]}\end{array}$ & Prospective & $\begin{array}{l}\text { Mixed } \\
\text { population }\end{array}$ & $\begin{array}{l}\text { Change at } 1 \text { through to } 105 \text { months } \\
\text { post onset }\end{array}$ & 20 & 61.5 -improvement & $\begin{array}{l}\text { Kinetic } \\
\text { perimetry }\end{array}$ \\
\hline $\begin{array}{l}2007 ; \\
\text { Kedar et } \\
\text { al. [55] }\end{array}$ & Retrospective & $\begin{array}{l}\text { Mixed } \\
\text { population }\end{array}$ & Median 3 days post onset & 852 & $\begin{array}{l}\text { Congruous hemianopia: } \\
38.1 \text { - improvement } \\
58.5 \text { - stable field } \\
3.4 \text { - deteriorated } \\
\text { Incongruous hemianopia: } \\
39.6 \text { - improvement } \\
41.5 \text { - stable field } \\
18.9 \text { - deteriorated }\end{array}$ & $\begin{array}{l}\text { Perimetry } \\
\text { Central } 30 \text { or } \\
24 \text { degrees }\end{array}$ \\
\hline $\begin{array}{l}2013 c ; \\
\text { Rowe et } \\
\text { al. [21] }\end{array}$ & Prospective & $\begin{array}{l}\text { Stroke } \\
\text { survivors with } \\
\text { suspected } \\
\text { visual } \\
\text { impairment }\end{array}$ & Variable over 2 weeks to 6 months & 915 & $\begin{array}{l}7.5-\text { full recovery } \\
39.2 \text { - partial recovery } \\
1-\text { deterioration } \\
52.3 \text { - static }\end{array}$ & $\begin{array}{l}\text { Confrontation } \\
\text { Static } \\
\text { perimetry } \\
\text { Kinetic } \\
\text { perimetry }\end{array}$ \\
\hline
\end{tabular}


Table 8. Recovery of eye movement deficits

\begin{tabular}{|c|c|c|c|c|c|c|}
\hline Study & Design & Population & $\begin{array}{l}\text { Time of vision } \\
\text { assessment }\end{array}$ & $\begin{array}{l}\text { Sample size } \\
(n=)\end{array}$ & Prevalence of visual issue (\%) & Assessment \\
\hline $\begin{array}{l}\text { 1982; De } \\
\text { Renzi } \\
\text { et al. [62] }\end{array}$ & Prospective & General stroke & $\begin{array}{l}\text { Follow-up every } 3-4 \\
\text { days for } 2 \text { weeks post } \\
\text { onset }\end{array}$ & 91 & $\begin{array}{l}8.6 \text { days - mean duration to } \\
\text { improvement with left stroke } \\
14.9 \text { - mean duration to improvement } \\
\text { with right stroke }\end{array}$ & NIHSS \\
\hline $\begin{array}{l}\text { 1987; } \\
\text { Freeman \& } \\
\text { Rudge [35] }\end{array}$ & Prospective & General stroke & $\begin{array}{l}\text { Up to } 12 \text { months post } \\
\text { onset }\end{array}$ & 76 & $\begin{array}{l}7 \text { - full improvement } \\
50 \text { - partial improvement } \\
43 \text { - stable } \\
92-\text { improvement in stereoacuity within } \\
1 \text { month }\end{array}$ & Orthoptic \\
\hline $\begin{array}{l}\text { 2011; Rowe } \\
\text { et al. [18] }\end{array}$ & Prospective & $\begin{array}{l}\text { Stroke survivors } \\
\text { with suspected } \\
\text { visual impairment }\end{array}$ & $\begin{array}{l}\text { Variable over } 2 \text { weeks } \\
\text { to } 6 \text { months }\end{array}$ & 915 & $\begin{array}{l}\text { Cranial nerve palsy: } \\
22.5 \text { - full improvement } \\
43 \text { - partial improvement } \\
3.5 \text { - deterioration } \\
\text { Nystagmus: } \\
42 \text { - partial improvement } \\
24 \text { - stable } \\
\text { Gaze palsy: } \\
4 \text { - full improvement } \\
66 \text { - partial improvement } \\
30 \text { - stable }\end{array}$ & Orthoptic \\
\hline $\begin{array}{l}\text { 2013; Ali et al. } \\
\text { [30] }\end{array}$ & Prospective & $\begin{array}{l}\text { Stroke trial } \\
\text { database }\end{array}$ & $\begin{array}{l}\text { Baseline, } 30 \text { days and } \\
90 \text { days }\end{array}$ & $\begin{array}{l}11900 \text { at } \\
\text { baseline } \\
4965 \text { at follow- } \\
\text { up }\end{array}$ & $\begin{array}{l}\text { Complete gaze palsy: } \\
\text { - at } 30 \text { days } \\
\text { Versus } 14.5 \% \text { at baseline } \\
\text { Partial gaze palsy: } \\
9 \text { - at } 30 \text { days } \\
\text { Versus } 31 \% \text { at baseline }\end{array}$ & $\begin{array}{l}\text { NIHSS } \\
\text { Confrontation }\end{array}$ \\
\hline
\end{tabular}


Table 9. Recovery of central vision deficit

\begin{tabular}{|c|c|c|c|c|c|c|}
\hline Study & Design & Population & Time of vision assessment & Sample size $(n=)$ & Prevalence of visual issue (\%) & Assessment \\
\hline $\begin{array}{l}\text { 1987; Freeman } \\
\text { \& Rudge [35] }\end{array}$ & $\begin{array}{l}\text { Prospective } \\
\text { observation }\end{array}$ & General stroke & Median within 1 week of onset & 247 & $71-$ improvement & $\begin{array}{l}\text { Medical } \\
\text { Orthoptic }\end{array}$ \\
\hline $\begin{array}{l}\text { 2011; Rowe et } \\
\text { al. [19] }\end{array}$ & Prospective & $\begin{array}{l}\text { Stroke survivors } \\
\text { with suspected } \\
\text { visual impairment }\end{array}$ & $\begin{array}{l}\text { Variable over } 2 \text { weeks to } 6 \\
\text { months }\end{array}$ & 915 & $\begin{array}{l}10.5 \text { - full improvement } \\
43.4 \text { - partial improvement } \\
44.7 \text { - stable } \\
1.3 \text { - deteriorated }\end{array}$ & Orthoptic \\
\hline
\end{tabular}

Table 10. Recovery of visual perceptual impairment

\begin{tabular}{|c|c|c|c|c|c|c|}
\hline Study & Design & Population & Time of vision assessment & Sample size $(n=)$ & $\begin{array}{l}\text { Prevalence of visual } \\
\text { issue (\%) }\end{array}$ & Assessment \\
\hline $\begin{array}{l}\text { 1987; Freeman \& } \\
\text { Rudge [35] }\end{array}$ & Prospective & General stroke & Up to 4 months post onset & 247 & $\begin{array}{l}\text { Visual neglect: } \\
29 \text { - complete recovery } \\
57 \text { - stable }\end{array}$ & $\begin{array}{l}\text { Medical } \\
\text { Orthoptic }\end{array}$ \\
\hline $\begin{array}{l}\text { 1998; Cassidy et } \\
\text { al. [79] }\end{array}$ & Prospective & $\begin{array}{l}\text { General stroke with } \\
\text { left hemisphere } \\
\text { lesions excluded }\end{array}$ & Monthly follow-up & 66 & $\begin{array}{l}9.1-\text { visual neglect at } 3 \\
\text { months } \\
\text { Versus } 40.9 \% \text { at baseline }\end{array}$ & $\begin{array}{l}\text { Behavioural } \\
\text { inattention } \\
\text { test }\end{array}$ \\
\hline $\begin{array}{l}\text { 2004; Farne et al. } \\
\text { [88] }\end{array}$ & Prospective & R hemisphere only & $\begin{array}{l}\text { Follow-up at } 2 \text { weeks and } 3 \\
\text { months post onset }\end{array}$ & $\begin{array}{l}33 \text { at baseline } \\
8 \text { at } 3 \text { months }\end{array}$ & $\begin{array}{l}43-\text { improvement at } 2 \\
\text { weeks [9-full] } \\
63-\text { improvement at } 3 \\
\text { months }\end{array}$ & $\begin{array}{l}\text { Behavioural } \\
\text { inattention } \\
\text { test }\end{array}$ \\
\hline $\begin{array}{l}\text { 2007; Poggel et al. } \\
\text { [89] }\end{array}$ & Prospective & $\begin{array}{l}\text { Post-geniculate } \\
\text { lesions }\end{array}$ & $\begin{array}{l}\text { Mean } 36 \text { months (7-189 } \\
\text { months), up to } 6 \text { months } \\
\text { follow-up. }\end{array}$ & 19 & $\begin{array}{l}\text { Visual hallucinations } \\
\text { persisted for several } \\
\text { days/weeks and then } \\
\text { gradually subsided }\end{array}$ & Interview \\
\hline $\begin{array}{l}\text { 2007; Poggel et al. } \\
\text { [89] }\end{array}$ & $\begin{array}{l}\text { Retrospective } \\
\text { questionnaire }\end{array}$ & Mixed population & Up to 6 months follow-up & 121 & Mean duration of 28 days & Questionnaire \\
\hline 2013; Ali et al. [30] & Prospective & $\begin{array}{l}\text { Stroke trial } \\
\text { database }\end{array}$ & $\begin{array}{l}\text { Baseline, } 30 \text { days and } 90 \\
\text { days }\end{array}$ & $\begin{array}{l}11900 \text { at baseline } \\
4965 \text { at follow-up }\end{array}$ & $\begin{array}{l}0.6 \text { - visual neglect at } 90 \\
\text { days } \\
\text { Versus } 27.7 \% \text { at baseline }\end{array}$ & $\begin{array}{l}\text { NIHSS } \\
\text { Confrontation }\end{array}$ \\
\hline
\end{tabular}


Accuracy of non-specialist vision assessments and accuracy of screening tools and scores is likely to impact on reported prevalence figures. Where basic screening is undertaken, it is possible to miss subtle visual problems whose ocular signs are not included in the screening assessment. Thus there is the potential for underdiagnoses when the assessment is performed by the stroke team rather than an eye team specialist or where screening tools are used which only measure specific features of vision, e.g. detection of hemianopia or horizontal gaze defects only as with the NIHSS, or reliance on basic confrontation assessment rather than detailed confrontation or perimetry assessment.

Studies that report sub populations of stroke survivors are also prone to reporting bias for visual problems. Despite large sample sizes in studies that have included sub populations of stroke survivors, such as the VIS study of those already suspected of having visual impairment or studies of clinical trial databases, these studies are unlikely to be representative of the general stroke population [6, 30]. These estimates are potential under- or over-representations of the true prevalence of visual problems across all stroke survivors.

The time of the baseline assessment is crucial for studies tracking the recovery of visual impairment. If the baseline assessment is delayed, complete or partial recovery may have already taken place. Furthermore, it has not yet been accurately established at what time point recovery of each visual problem following stroke can be expected. If a study only has short period of follow-up, recovery could continue after the participant has completed the study. Both factors result in under-estimation of recovery of strokerelated visual impairment.

Future studies are required to establish the incidence for post-stroke visual impairment in the early acute period within the first week of onset. Such studies should involve a full stroke cohort with no exclusions so that visual impairment rates are comprehensively evaluated. These patients require follow-up at regular time intervals to plot change in visual impairment over the first week, first month and longer term after stroke onset to provide information on trajectory of improvement, if any, and rates for full, partial or no recovery. At baseline and follow-up visits, full specialist assessment is required such that subtle visual deficits that can cause visual impairment are not missed.

\section{CONCLUSIONS}

The literature currently available for review does not include any studies whose primary aim was to determine incidence or prevalence of visual impairment post stroke. Thus, this review can only provide estimates of prevalence for individual stroke related visual problems. The estimation of the overall prevalence of visual impairment was approximately $65 \%$ at baseline assessment. A reduction to approximately $20 \%$ is seen by three month post stroke, due to factors such as recovery, adaptation and death. The figures reported cover a wide range of prevalence for each visual problem. A variety of factors may be the cause of this wide range of figures including; the different study aims, research methods used, baseline assessments being conducted at different time points and different methods assessment. The prevalence is reported as being highest for eye movement defects, visual field loss and visual inattention. The existing literature regarding the recovery of visual problems following stroke is scarce for both individual deficits and overall visual recovery. Further prospective studies are required to establish the incidence of post-stroke visual impairment, the prevalence at various time periods post stroke and trajectory of improvement.

\section{ETHICAL APPROVAL}

It is not applicable.

\section{ACKNOWLEDGEMENTS}

This review was supported by funding from the Stroke Association and Thomas Pocklington Trust. We acknowledge the advice received from Professor Rumona Dickson, University of Liverpool, and $\mathrm{Dr}$ Alex Pollock, Glasgow Caledonian University.

\section{COMPETING INTERESTS}

Authors have declared that no competing interests exist.

\section{REFERENCES}

1. Pollock A, et al. Interventions for disorders of eye movement in patients with stroke. Cochrane Database of Systematic Reviews. 2011;10. 
2. Pollock $A$, et al. Interventions for visual field defects in patients with stroke. Cochrane Database of Systematic Reviews. 2011;10.

3. Pollock A, et al. Interventions for agerelated visual problems in patients with stroke. Cochrane Database of Systematic Reviews. 2012;3.

4. Bowen A et al. Cognitive rehabilitation for spatial neglect following stroke. Cochrane Database of Systematic Reviews. 2013;7.

5. Jones SA, Shinton RA. Improving outcome in stroke patients with visual problems. Age \& Ageing. 2006;35(6):560-565.

6. Rowe $\mathrm{F}$ et al. Visual impairment following stroke: do stroke patients require vision assessment? Age \& Ageing. 2009;38(2): 188-193.

7. Granger $\mathrm{CV}$ et al. Functional assessment scales: A study of persons after stroke. Archives of Physical Medicine \& Rehabilitation. 1993;74(2):133-138.

8. Nelles $\mathrm{G}$, et al. Compensatory visual field training for patients with hemianopia after stroke. Neuroscience Letters. 2001;306(3): 189-192.

9. Ramrattan RS, et al. Prevalence and causes of visual field loss in the elderly and associations with impairment in daily functioning: The rotterdam study. Archives of Ophthalmology. 2001;119(12): 17881794.

10. West CG, et al. Is vision function related to physical functional ability in older adults? Journal of the American Geriatrics Society. 2002;50(1):136-145.

11. Tsai SY, et al. Association between visual impairment and depression in the elderly. Journal of the Formosan Medical Association. 2003;102(2):86-90.

12. Maberley DAL, et al. The prevalence of low vision and blindness in Canada. Eye. 2006;20(3):341-346.

13. Hyman $L$, et al. Prevalence and causes of visual impairment in the Barbados eye study1. Ophthalmology. 2001;108(10): 1751-1756.

14. Hsu WM, et al. Prevalence and causes of visual impairment in an elderly Chinese population in Taiwan1: The shihpai eye study. Ophthalmology. 2004;111(1):62-69.

15. Rodriguez J, et al. Causes of blindness and visual impairment in a populationbased sample of U.S. Hispanics. Ophthalmology. 2002;109(4):737-743.
16. Klein R, Klein BEK, Lee KE. Changes in visual acuity in a population. Ophthalmology. 1996;103(8):1169-1178.

17. Marmamula $\mathrm{S}$, et al. A cross-sectional study of visual impairment in elderly population in residential care in the South Indian state of Andhra Pradesh: A crosssectional study. BMJ Open. 2013;3(3).

18. Rowe F. Prevalence of ocular motor cranial nerve palsy and associations following stroke. Eye. $2011 ; 25(7): 881-887$.

19. Rowe $F$, et al. Reading difficulty after stroke: Ocular and non ocular causes. International Journal of Stroke. 2011;6(5): 404-411.

20. Pollock $A$, et al. Interventions for visual field defects in patients with stroke. Stroke. 2012;43(4):e37-e38.

21. Rowe $\mathrm{F}$ et al. A prospective profile of visual field loss following stroke: Prevalence, type, rehabilitation and outcome. Bio Med Research International; 2013.

22. Rowe F, et al. Profile of gaze dysfunction following cerebro vascular accident. ISRN Ophthalmology; 2013.

23. Rowe $\mathrm{F}$, et al. The profile of strabismus in stroke survivors. Eye. 2010;24(4):682-685.

24. Rowe F. Visual perceptual consequences of stroke. Strabismus. 2009;17(1):24-28.

25. University of York centre for reviews and dissemination. PROSPERO: International prospective register of systematic reviews. 2013;27:2015.

Available:http://www.crd.york.ac.uk/PROS PERO/

26. Von Elm E, et al. Strengthening the reporting of observational studies in epidemiology (STROBE): Guidelines for reporting observational studies. Plos Medicine. 2007;4(10):1623-1627.

27. Vandenbroucke JP, et al. Strengthening the reporting of observational studies in epidemiology (STROBE): Explanation and elaboration. Plos Medicine. 2007;4(10): 1628-1654.

28. Sanderson S, Tatt ID, Higgins JP. Tools for assessing quality and susceptibility to bias in observational studies in epidemiology: $A$ systematic review and annotated bibliography. International Journal of Epidemiology. 2007;36(3):666-676.

29. Boyle $\mathrm{MH}$, Guidelines for evaluating prevalence studies. Evidence-Based Mental Health. 1998;1(2):37-39.

30. Ali $\mathrm{M}$, et al. Recovery from poststroke visual impairment: Evidence from a clinical 
trials resource. Neurorehabilitation \& Neural Repair. 2013;27(2):133-141.

31. Gall SL, et al. Sex differences in presentation, severity, and management of stroke in a population-based study. Neurology. 2010;74(12):975-981.

32. Clisby C. Visual assessment of patients with cerebrovascular accident on the elderly care wards. British Orthoptic Journal. 1995;52:38-41.

33. Isaeff WB, Wallar PH, Duncan G. Ophthalmic findings in 322 patients with a cerebral vascular accident. Annals of Ophthalmology. 1974;6(10):1059-1064.

34. Trobe JD, Lorber ML, Schlezinger NS. Isolated homonymous hemianopia. A review of 104 cases. Archives of Ophthalmology. 1973;89(5):377-381.

35. Freeman CF, Rudge NB. The orthoptist's role in the management of stroke patients. in $6^{\text {th }}$ International Orthoptic Congress. Harrogate, UK; 1987.

36. Rowe F, et al. Symptoms of stroke-related visual impairment. Strabismus. 2013;21(2): 150-154.

37. Rothwell PM, External validity of randomised controlled trials: "To whom do the results of this trial apply?" The Lancet. 2005;365(9453):82-93.

38. Barrett KM, et al. Sex differences in stroke severity, symptoms, and deficits after firstever ischemic stroke. Journal of Stroke \& Cerebrovascular Diseases. 2007;16(1):3439.

39. Lyden $\mathrm{P}$, et al. Underlying structure of the national institutes of health stroke scale: Results of a factor analysis. Stroke. 1999; 30(11):2347-2354.

40. Jones WJ, Williams LS, Meschia JF, Validating the questionnaire for verifying stroke-Free status (Qvsfs) by neurological history and examination. Stroke. 2001; 32(10):2232-2236.

41. Gray CS, et al. Recovery of visual fields in acute stroke: Homonymous hemianopia associated with adverse prognosis. Age and Ageing. 1989;18(6):419-421 .

42. Searls DE, et al. Symptoms and signs of posterior circulation ischemia in the new England medical center posterior circulation registry. Archives of Neurology. 2012;69(3):346-351.

43. Tao WD, et al. Posterior versus anterior circulation infarction: How different are the neurological deficits? Stroke. 2012;43(8): 2060-2065.
44. Zhang $X$, et al. Homonymous hemianopias: Clinical-anatomic correlations in 904 cases. Neurology. 2006;66(6): 906-910.

45. Agrell BM, Dehlin OI, Dahlgren CJ. Neglect in elderly stroke patients: A comparison of five tests. Psychiatry and Clinical Neurosciences. 1997;51(5):295-300.

46. Cassidy TP, Bruce DW, Gray CS, Visual field loss after stroke: Confrontation and perimetry in the assessment of recovery. Journal of Stroke and Cerebrovascular Diseases. 2001;10(3):113-117.

47. Haerer AF. Visual field defects and the prognosis of stroke patients. Stroke. 1973; 4(2):163-168.

48. Benedetti MD, et al. Short term prognosis of stroke in a clinical series of 94 patients. The Italian Journal of Neurological Sciences. 1993;14(2):121-127.

49. Lawrence ES, et al. Estimates of the prevalence of acute stroke impairments and disability in a multiethnic population. Stroke. 2001;32(6):1279-1284.

50. $\mathrm{Ng} \mathrm{YS}$, et al. Clinical characteristics and rehabilitation outcomes of patients with posterior cerebral artery stroke. Archives of Physical Medicine and Rehabilitation. 2005;86(11):2138-2143.

51. Townend BS, et al. Perimetric homonymous visual field loss post-stroke. Journal of Clinical Neuroscience.2007;14(8):754-756.

52. Rathore SS, et al. Characterization of incident stroke signs and symptoms: Findings from the atherosclerosis risk in communities study. Stroke. 2002;33(11): 2718-2721.

53. Jerath NU, et al. Gender differences in presenting signs and symptoms of acute ischemic stroke: A population-based study. Gender Medicine. 2011;8(5):312-319.

54. Celesia GG, Brigell MG, Vaphiades MS, Hemianopic anosognosia. Neurology. 1997;49(1):88-97.

55. Kedar $\mathrm{S}$, et al. Congruency in homonymous hemianopia. American Journal of Ophthalmology. 2007;143(5):772-780.

56. Stone SP, Halligan PW, Greenwood RJ, The incidence of neglect phenomena and related disorders in patients with an acute right or left hemisphere stroke. Age \& Ageing. 1993;22(1):46-52.

57. Yap MHL, Loong SC, Nei IP. Eye signs in strokes. Annals of the Academy of Medicine Singapore. 1975;4(2):133-137. 
58. Fowler MS, et al. Squints and diplopia seen after brain damage. Journal of Neurology. 1996;243(1):86-90.

59. Maeshima $S$, et al. Functional outcome in patients with pontine infarction after acute rehabilitation. Neurological Sciences. 2012;33(4):759-764.

60. Su $\mathrm{CH}$, Young $\mathrm{YH}$, Clinical significance of pathological eye movements in diagnosing posterior fossa stroke. Acta OtoLaryngologica. 2013;133(9):916-923.

61. Siong $\mathrm{KH}$, et al. Prevalence of visual problems among stroke survivors in Hong Kong Chinese. Clinical and Experimental Optometry. 2014;97:433-441.

62. De Renzi E, et al. Conjugate gaze paresis in stroke patients with unilateral damage. An unexpected instance of hemispheric asymmetry. Archives of Neurology. 1982; 39(8):482-486.

63. Singer $\mathrm{OC}$, et al. Conjugate eye deviation in acute stroke: Incidence, hemispheric asymmetry, and lesion pattern. Stroke. 2006;37(11):2726-2732.

64. Lotery AJ, et al. Correctable visual impairment in stroke rehabilitation patients. Age and Ageing. 2000;29(3):221-222.

65. Siddique MAN, et al. Clinical presentation and epidemiology of stroke - A study of 100 cases. Journal of Medicine. 2009; 10(2):86-89.

66. Rowe $\mathrm{F}$, et al. The spectrum of nystagmus following cerebro-vascular accident. British and Irish Orthoptic Journal. 2008;5:22-25.

67. Baier B, Dieterich $M$. Incidence and anatomy of gaze-evoked nystagmus in patients with cerebellar lesions. Neurology. $2011 ; 76(4): 361-365$.

68. Akhtar $\mathrm{N}$, et al. Ischaemic posterior circulation stroke in State of Qatar. European Journal of Neurology. 2009; 16(9):1004-1009.

69. Rowe $\mathrm{F}$, et al. Visual impairment in stroke survivors: A prospective multi-centre trial. in $31^{\text {st }}$ European Strabismological Association. Mykonos, Greece; 2007.

70. Edwards DF, et al. Screening patients with stroke for rehabilitation needs: Validation of the post-stroke rehabilitation guidelines. Neurorehabilitation and Neural Repair. 2006;20(1):42-48.

71. Bulens C, et al. Spatial contrast sensitivity in unilateral cerebral ischaemic lesions involving the posterior visual pathway. Brain. 1989;112(Pt 2):507-520.
72. Dos Santos NA, Andrade SM. Visual contrast sensitivity in patients with impairment of functional independence after stroke. BMC Neurology. 2012;12:90.

73. Pedersen PM, et al. Hemi neglect in acute stroke--incidence and prognostic implications. The Copenhagen Stroke Study. American Journal of Physical Medicine \& Rehabilitation. 1997;76(2):122-127.

74. Appelros $P$, et al. Neglect and anosognosia after first-ever stroke: Incidence and relationship to disability. Journal of Rehabilitation Medicine. 2002; 34(5):215-220.

75. Linden $\mathrm{T}$, et al. Visual neglect and cognitive impairment in elderly patients late after stroke. Acta Neurologica Scandinavica. 2005;111(3):163-168.

76. Becker E, Karnath HO. Incidence of visual extinction after left versus right hemisphere stroke. Stroke. 2007;38(12):3172-3174.

77. Van Nes IJ, et al. Is visuospatial hemineglect really a determinant of postural control following stroke? An acute-phase study. Neurorehabilitation \& Neural Repair. 2009;23(6):609-614.

78. Lee $\mathrm{BH}$, et al. Neglect dyslexia: Frequency, association with other hemispatial neglects, and lesion localization. Neuropsychologia. 2009;47(3):704-710.

79. Cassidy TP, Lewis S, Gray CS. Recovery from visuospatial neglect in stroke patients. Journal of Neurology, Neurosurgery \& Psychiatry. 1998;64(4): 555-557.

80. Cassidy TP, et al. The association of visual field deficits and visuo-spatial neglect in acute right-hemisphere stroke patients. Age \& Ageing. 1999;28(3):257-260.

81. Shrestha GS, et al. Ocular-visual defect and visual neglect in stroke patients - A report from Kathmandu, Nepal. Journal of Optometry. 2012;5(1):43-49.

82. Beaudoin AJ, et al. Visuoperceptual deficits and participation in older adults after stroke. Australian Occupational Therapy Journal. 2013;60(4):260-266.

83. Yang $\mathrm{TH}$, et al. Topology of brainstem lesions associated with subjective visual vertical tilt. Neurology. 2014;82:1968-1975.

84. Chechlacz $M$, et al. The frequency and severity of extinction after stroke affecting different vascular territories. Neuropsychologia. 2014;54:11-17. 
85. Tiel K, Kolmel HW. Patterns of recovery from homonymous hemianopia subsequent to infarction in the distribution of the posterior cerebral artery. NeuroOphthalmology. 1991;11(1):33-39.

86. Schmielau FEK, Wong Jr. Recovery of visual fields in brain-lesioned patients by reaction perimetry treatment. Journal of Neuro Engineering and Rehabilitation. 2007;4.

87. Zhang $\mathrm{X}$, et al. Natural history of homonymous hemianopia. Neurology. 2006;66: 901-905.
88. Farne $A$, et al. Patterns of spontaneous recovery of neglect and associated disorders in acute right brain-damaged patients. Journal of Neurology, Neurosurgery \& Psychiatry. 2004;75(10):14011410.

89. Poggel DA, et al. Visual hallucinations during spontaneous and training-induced visual field recovery. Neuropsychologia. 2007;45(11):2598-2607. 


\section{APPENDIX}

\section{Appendix 1 - PRISMA 2009 Checklist}

\begin{tabular}{|c|c|c|c|}
\hline \multicolumn{4}{|l|}{ Title } \\
\hline Title & 1 & $\begin{array}{l}\text { Identify the report as a systematic review, meta-analysis, or } \\
\text { both. }\end{array}$ & 1 \\
\hline \multicolumn{4}{|c|}{ e } \\
\hline $\begin{array}{l}\text { Structured } \\
\text { summary }\end{array}$ & 2 & $\begin{array}{l}\text { Provide a structured summary including, as applicable: } \\
\text { background; objectives; data sources; study eligibility } \\
\text { criteria, participants, and interventions; study appraisal and } \\
\text { synthesis methods; results; limitations; conclusions and } \\
\text { implications of key findings; systematic review registration } \\
\text { number. }\end{array}$ & 1 \\
\hline \multicolumn{4}{|l|}{ Introduction } \\
\hline Rationale & 3 & $\begin{array}{l}\text { Describe the rationale for the review in the context of what is } \\
\text { already known. }\end{array}$ & 2 \\
\hline Objectives & 4 & $\begin{array}{l}\text { Provide an explicit statement of questions being addressed } \\
\text { with reference to participants, interventions, comparisons, } \\
\text { outcomes, and study design (PICOS). }\end{array}$ & 2 \\
\hline \multicolumn{4}{|l|}{ Methods } \\
\hline $\begin{array}{l}\text { Protocol and } \\
\text { registration }\end{array}$ & 5 & $\begin{array}{l}\text { Indicate if a review protocol exists, if and where it can be } \\
\text { accessed (e.g., Web address), and, if available, provide } \\
\text { registration information including registration number. }\end{array}$ & $\mathrm{N} / \mathrm{A}$ \\
\hline $\begin{array}{l}\text { Eligibility } \\
\text { criteria }\end{array}$ & 6 & $\begin{array}{l}\text { Specify study characteristics (e.g., PICOS, length of follow- } \\
\text { up) and report characteristics (e.g., years considered, } \\
\text { language, publication status) used as criteria for eligibility, } \\
\text { giving rationale. }\end{array}$ & $2-3$ \\
\hline $\begin{array}{l}\text { Information } \\
\text { sources }\end{array}$ & 7 & $\begin{array}{l}\text { Describe all information sources (e.g., databases with dates } \\
\text { of coverage, contact with study authors to identify additional } \\
\text { studies) in the search and date last searched. }\end{array}$ & Appendix 2 \\
\hline Search & 8 & $\begin{array}{l}\text { Present full electronic search strategy for at least one } \\
\text { database, including any limits used, such that it could be } \\
\text { repeated. }\end{array}$ & Appendix 2 \\
\hline $\begin{array}{l}\text { Study } \\
\text { selection }\end{array}$ & 9 & $\begin{array}{l}\text { State the process for selecting studies (i.e., screening, } \\
\text { eligibility, included in systematic review, and, if applicable, } \\
\text { included in the meta-analysis). }\end{array}$ & $2-3$ \\
\hline $\begin{array}{l}\text { Data collection } \\
\text { process }\end{array}$ & 10 & $\begin{array}{l}\text { Describe method of data extraction from reports (e.g., } \\
\text { piloted forms, independently, in duplicate) and any } \\
\text { processes for obtaining and confirming data from } \\
\text { investigators. }\end{array}$ & 4 \\
\hline Data items & 11 & $\begin{array}{l}\text { List and define all variables for which data were sought (e.g., } \\
\text { PICOS, funding sources) and any assumptions and } \\
\text { simplifications made. }\end{array}$ & $3-4$ \\
\hline $\begin{array}{l}\text { Risk of bias in } \\
\text { individual } \\
\text { studies }\end{array}$ & 12 & $\begin{array}{l}\text { Describe methods used for assessing risk of bias of } \\
\text { individual studies (including specification of whether this was } \\
\text { done at the study or outcome level), and how this } \\
\text { information is to be used in any data synthesis. }\end{array}$ & $\begin{array}{l}4 \\
\text { Appendix } 5\end{array}$ \\
\hline $\begin{array}{l}\text { Summary } \\
\text { measures }\end{array}$ & 13 & $\begin{array}{l}\text { State the principal summary measures (e.g., risk ratio, } \\
\text { difference in means). }\end{array}$ & $\mathrm{N} / \mathrm{A}$ \\
\hline $\begin{array}{l}\text { Synthesis of } \\
\text { results }\end{array}$ & 14 & $\begin{array}{l}\text { Describe the methods of handling data and combining } \\
\text { results of studies, if done, including measures of consistency }\end{array}$ & $\mathrm{N} / \mathrm{A}$ \\
\hline
\end{tabular}




\begin{tabular}{|c|c|c|c|}
\hline \multicolumn{4}{|c|}{$\left(\right.$ e.g., $\left.I^{2}\right)$ for each meta-analysis. } \\
\hline $\begin{array}{l}\text { Risk of bias } \\
\text { across studies }\end{array}$ & 15 & $\begin{array}{l}\text { Specify any assessment of risk of bias that may affect the } \\
\text { cumulative evidence (e.g., publication bias, selective } \\
\text { reporting within studies). }\end{array}$ & 4 \\
\hline $\begin{array}{l}\text { Additional } \\
\text { analyses }\end{array}$ & 16 & $\begin{array}{l}\text { Describe methods of additional analyses (e.g., sensitivity or } \\
\text { subgroup analyses, meta-regression), if done, indicating } \\
\text { which were pre-specified. }\end{array}$ & $\mathrm{N} / \mathrm{A}$ \\
\hline \multicolumn{4}{|c|}{ ( } \\
\hline $\begin{array}{l}\text { Study } \\
\text { selection }\end{array}$ & 17 & $\begin{array}{l}\text { Give numbers of studies screened, assessed for eligibility, } \\
\text { and included in the review, with reasons for exclusions at } \\
\text { each stage, ideally with a flow diagram. }\end{array}$ & 4, Appendix 3 \\
\hline $\begin{array}{l}\text { Study } \\
\text { characteristics }\end{array}$ & 18 & $\begin{array}{l}\text { For each study, present characteristics for which data were } \\
\text { extracted (e.g., study size, PICOS, follow-up period) and } \\
\text { provide the citations. }\end{array}$ & $\begin{array}{l}\text { Tables } 1 \text { to } \\
10\end{array}$ \\
\hline $\begin{array}{l}\text { Risk of bias } \\
\text { within studies }\end{array}$ & 19 & $\begin{array}{l}\text { Present data on risk of bias of each study and, if available, } \\
\text { any outcome level assessment (see item 12). }\end{array}$ & $\begin{array}{l}4 \\
\text { Appendix } 5\end{array}$ \\
\hline $\begin{array}{l}\text { Results of } \\
\text { individual } \\
\text { studies }\end{array}$ & 20 & $\begin{array}{l}\text { For all outcomes considered (benefits or harms), present, for } \\
\text { each study: (a) simple summary data for each intervention } \\
\text { group (b) effect estimates and confidence intervals, ideally } \\
\text { with a forest plot. }\end{array}$ & $\begin{array}{l}\text { Tables } 1 \text { to } \\
10\end{array}$ \\
\hline $\begin{array}{l}\text { Synthesis of } \\
\text { results }\end{array}$ & 21 & $\begin{array}{l}\text { Present results of each meta-analysis done, including } \\
\text { confidence intervals and measures of consistency. }\end{array}$ & $\mathrm{N} / \mathrm{A}$ \\
\hline $\begin{array}{l}\text { Risk of bias } \\
\text { across studies }\end{array}$ & 22 & $\begin{array}{l}\text { Present results of any assessment of risk of bias across } \\
\text { studies (see Item 15). }\end{array}$ & 4 \\
\hline $\begin{array}{l}\text { Additional } \\
\text { analysis }\end{array}$ & 23 & $\begin{array}{l}\text { Give results of additional analyses, if done (e.g., sensitivity } \\
\text { or subgroup analyses, meta-regression [see Item 16]). }\end{array}$ & $\mathrm{N} / \mathrm{A}$ \\
\hline \multicolumn{4}{|l|}{ Discussion } \\
\hline $\begin{array}{l}\text { Summary of } \\
\text { evidence }\end{array}$ & 24 & $\begin{array}{l}\text { Summarize the main findings including the strength of } \\
\text { evidence for each main outcome; consider their relevance to } \\
\text { key groups (e.g., healthcare providers, users, and policy } \\
\text { makers). }\end{array}$ & $5-24$ \\
\hline Limitations & 25 & $\begin{array}{l}\text { Discuss limitations at study and outcome level (e.g., risk of } \\
\text { bias), and at review-level (e.g., incomplete retrieval of } \\
\text { identified research, reporting bias). }\end{array}$ & $24-25$ \\
\hline Conclusions & 26 & $\begin{array}{l}\text { Provide a general interpretation of the results in the context } \\
\text { of other evidence, and implications for future research. }\end{array}$ & 25 \\
\hline \multicolumn{4}{|l|}{ Funding } \\
\hline Funding & 27 & $\begin{array}{l}\text { Describe sources of funding for the systematic review and } \\
\text { other support (e.g., supply of data); role of funders for the } \\
\text { systematic review. }\end{array}$ & 25 \\
\hline
\end{tabular}




\section{Appendix 2. Search Options and Search Terms}

\section{Databases:}

- Cochrane Stroke Group Trials Register

- The Cochrane Eyes and Vision Group Trials Register

- The Cochrane Central Register of Controlled Trials (CENTRAL) (The Cochrane Library, latest issue);

- MEDLINE (1950 to April 2015);

- EMBASE (1980 to April 2015);

- CINAHL (1982 to April 2015);

- $\operatorname{AMED}(1985$ to April 2015);

- PsycINFO (1967 April 2015);

- Dissertations \& Theses (PQDT) database (1861 to April 2015);

- British Nursing Index (1985 to April 2015);

- PsycBITE (Psychological Database for Brain Impairment Treatment Efficacy, www.psycbite.com).

\section{Registers:}

- ClinicalTrials.gov (http://clinicaltrials.gov/);

- Current Controlled Trials (www.controlledtrials. com);

- Trials Central (www.trialscentral.org);

- Health Service Research Projects in Progress (wwwcf.nlm.nih.gov/hsr project/home proj.cfm);

- National Eye Institute Clinical Studies Database (http://clinicalstudies.info.nih.gov/cgi/protinstitute.cgi?NEl.0.html)

- British and Irish Orthoptic Journal, Australian Orthoptic Journal, and proceedings of the European Strabismological Association (ESA), International Strabismological Association (ISA), International Orthoptic Association (IOA) (http://pcwww.liv.ac.uk/ rowef/index files/Page646.htm)

- Proceedings of Association for Research in Vision and Ophthalmology (www.arvo.org);

Terms:

\begin{tabular}{|l|l|}
\hline Cerebrovascular disorders/ & Eye Movements/ \\
Brain ischaemia/ & Eye/ \\
Intracranial Arterial Disease & Eye Disease/ \\
Intracranial Arteriovenous Malformations/ & Visually Impaired Persons/ \\
"Intracranial Embolism and Thrombosis*/ & Vision Disorders/ \\
Stroke/ & Blindness/ \\
& Diplopia/ \\
& Vision, Binocular/ \\
& Vision, Monocular/ \\
& Visual Acuity/ \\
& Visual Fields/ \\
& Vision, Low/ \\
\hline
\end{tabular}




\begin{tabular}{|l|l|}
\hline & Ocular Motility Disorders/ \\
& Blindness, Cortical/ \\
Hemianopsia/ & Abducens Nerve Diseases/ \\
& Abducens Nerve/ \\
& Oculomotor Nerve/ \\
& Trochlear Nerve/ \\
& Visual Perception/ \\
& Nystagmus \\
& strabismus \\
& smooth pursuits \\
& saccades depth perception \\
& stereopsis gaze disorder \\
internuclear opthalmoplegia & Parinaud's syndrome \\
& Weber's syndrome \\
& skew deviation \\
& conjugate deviation oscillopsia \\
visual tracking agnosia hallucinations & OR \\
\hline AR & \\
\hline AND \\
\hline
\end{tabular}




\section{Appendix 3. Flowchart of Pathway for Inclusion of Articles}

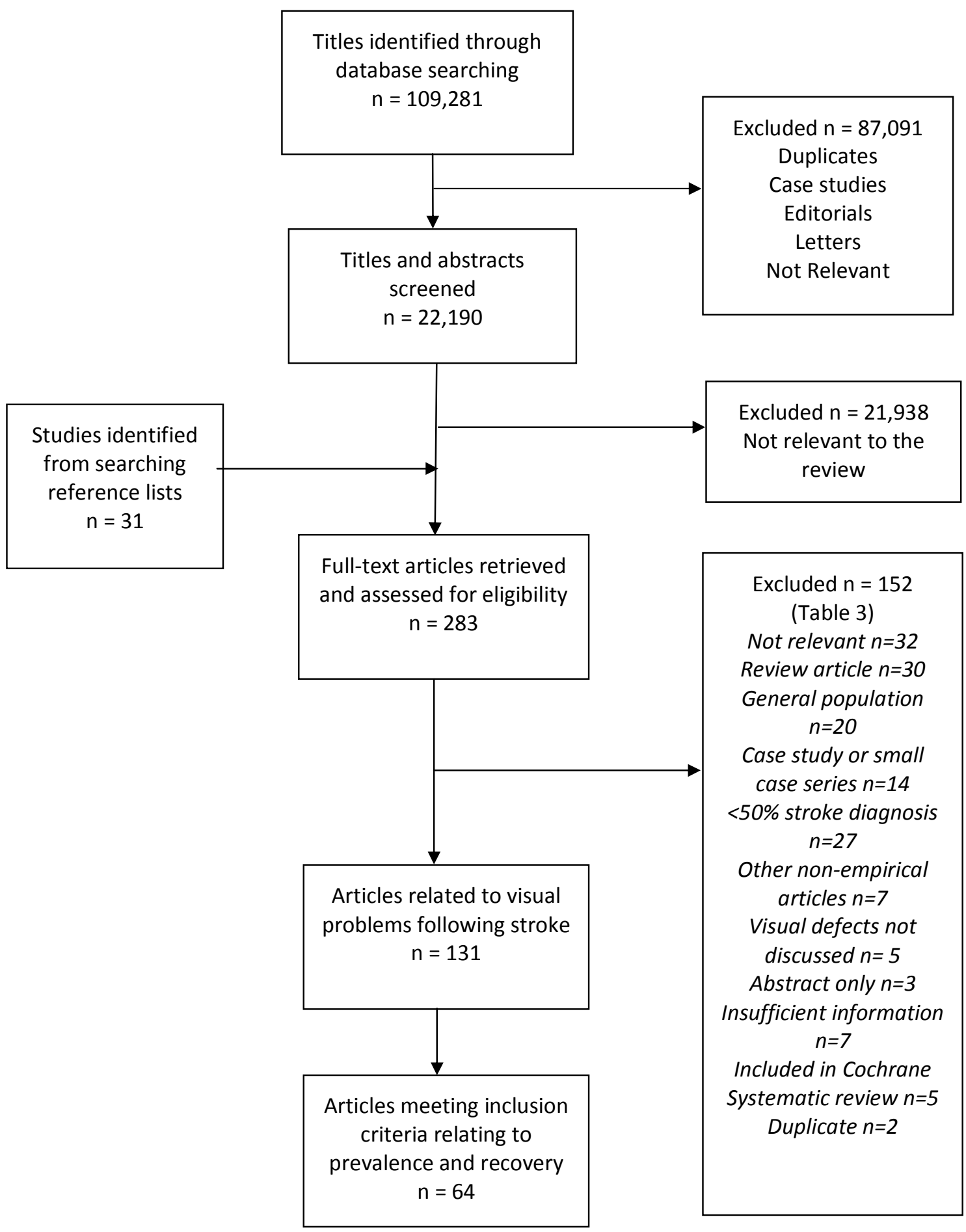




\section{Appendix 4. Excluded Articles}

\begin{tabular}{|c|c|}
\hline Study & Reason for exclusion \\
\hline Ajina and Kennard, 2012 & Review article \\
\hline Al-Khayat et al., 2005 & No stroke patients included \\
\hline Anderson and Rizzo, 1994 & Case report \\
\hline Anderson and Rizzo, 1995 & Review article \\
\hline Baier at al., 2010 & Not relevant to the review - preselected cases \\
\hline Barker et al., 2012 & $\begin{array}{l}\text { Not relevant to the review - assessment of } \\
\text { neuropsychology }\end{array}$ \\
\hline Barnes et al., 2006 & Unable to distinguish number of stroke patients \\
\hline Barrett, 2009 & Review article \\
\hline Bartolomei et al., 1998 & No stroke patients included \\
\hline Beran and Murphy-Lavoie, 2009 & Not related to stroke \\
\hline Beck and Harris, 1994 & Not related to stroke - general population \\
\hline Behrmann et al., 2004 & $\begin{array}{l}\text { Not relevant to the review - addresses different } \\
\text { types of search patterns in neglect }\end{array}$ \\
\hline Biousse et al., 1998 & Only reported on three patients \\
\hline Blythe et al., 1987 & $\begin{array}{l}\text { Not relevant to the review - preselected cases } \\
\text { assessed for blindsight }\end{array}$ \\
\hline Bodis-Wollner and Diamond, 1973 & $\begin{array}{l}\text { Unable to establish the proportion of participants } \\
\text { were post-stroke, participants reported to have } \\
\text { cerebral lesions. }\end{array}$ \\
\hline Bodis-Wollner and Diamond, 1976 & $\begin{array}{l}\text { Unable to establish the proportion of participants } \\
\text { were post-stroke, participants reported to have } \\
\text { cerebral lesions. }\end{array}$ \\
\hline Bombois et al., 2007 & Stroke patients excluded \\
\hline Bronstein et al., 1990 & $\begin{array}{l}\text { Unable to establish the proportion of participants } \\
\text { were post-stroke }\end{array}$ \\
\hline Brown Jr et al., 1998 & A general population sampled \\
\hline Brunette, 1967 & Review article \\
\hline Bulsara et al., 2007 & No stroke patients included \\
\hline Bunce and Wormald, 2008 & A general population sampled \\
\hline Bunce et al., 2010 & A general population sampled \\
\hline Büttner and Grundei, 1995 & Sample included $50 \%$ or fewer stroke patients \\
\hline Buxbaum et al., 2008 & $\begin{array}{l}\text { Not relevant to the review - performance on } \\
\text { wheelchair navigation }\end{array}$ \\
\hline Caneman et al., 1992 & $\begin{array}{l}\text { Not relevant to the review - performance on maze } \\
\text { test }\end{array}$ \\
\hline Carlow and Bicknell, 1981 & Review article \\
\hline Carman-Merrifield, 2005 & Review article \\
\hline Cheek et al., 1965 & Sample included $50 \%$ or fewer stroke patients \\
\hline Cheung et al., 2008 & $\begin{array}{l}\text { Not relevant to the review - discussed retinal } \\
\text { pathology }\end{array}$ \\
\hline Chia et al., 2004 & A general population sampled \\
\hline Ciuffreda et al., 2006 & Sample included $50 \%$ or fewer stroke patients \\
\hline Ciuffreda et al., 2007 & Sample included $50 \%$ or fewer stroke patients \\
\hline
\end{tabular}




\begin{tabular}{|c|c|}
\hline Clenet, 2011 & Case study \\
\hline Cockburn, 1983 & A general population sampled \\
\hline Colombo et al., 1981 & $\begin{array}{l}\text { Not relevant to the review - preselected cases from } \\
\text { a larger cohort }\end{array}$ \\
\hline Cooper, 1971 & Not relevant to stroke \\
\hline Cooper et al., 2012 & Only reported on two patients \\
\hline Crews et al., 2006 & Sample included $50 \%$ or fewer stroke patients \\
\hline Danta et al., 1978 & Sample included $50 \%$ or fewer stroke patients \\
\hline Das et al., 2007 & Review article \\
\hline Dennis et al., 1990 & $\begin{array}{l}\text { Not relevant to the review - transient ischaemic } \\
\text { attacks }\end{array}$ \\
\hline Di Legge et al., 2004 & Correspondence to the editor \\
\hline Dulli et al., 1998 & No reference to visual problems \\
\hline François, 1975 & Review article \\
\hline Fraser et al., 2011 & Review article \\
\hline Galanth et al., 2014 & Visual problems of stroke patients not discussed \\
\hline Gállego et al., 2008 & Review article \\
\hline Gamio and Melek, 2003 & Case report \\
\hline George et al., 2011 & Protocol article \\
\hline Georgiadis et al., 1999 & No reference to visual problems \\
\hline Gilhotra et al., 2002 & A general population sampled \\
\hline Gilhotra et al., 2002 & A general population sampled \\
\hline Giroud et al., 1994 & $\begin{array}{l}\text { Not relevant to review - focused on seizures after } \\
\text { stroke }\end{array}$ \\
\hline Globe et al., 2005 & A general population sampled \\
\hline Goldstein and Simel, 2005 & Review article \\
\hline Good et al., 2001 & Not relevant to review - paediatric population \\
\hline Gottlieb and Miesner, 2004 & Review article \\
\hline Grunda et al., 2013 & Review article \\
\hline Guenther et al., 2009 & $\begin{array}{l}\text { Not relevant to the review - evaluating prediction } \\
\text { model }\end{array}$ \\
\hline Habekost and Starrfelt, 2006 & Case report \\
\hline Hankey, 1997 & A general population sampled \\
\hline Hofman et al., 2007 & $\begin{array}{l}\text { A general population sampled and study protocol } \\
\text { update }\end{array}$ \\
\hline Hofman et al., 2011 & $\begin{array}{l}\text { A general population sampled and study protocol } \\
\text { update }\end{array}$ \\
\hline Horton, 2005 & Editorial \\
\hline Howard et al., 2006 & $\begin{array}{l}\text { Unable to establish the proportion of participants } \\
\text { were post-stroke }\end{array}$ \\
\hline Jagger et al., 1989 & No stroke patients included \\
\hline Jarvis et al., 2012 & $\begin{array}{l}\text { Not relevant to the review - information provided to } \\
\text { the stroke team }\end{array}$ \\
\hline Jensen et al., 2009 & Case study \\
\hline Jin et al., 2010 & No stroke patients included \\
\hline Jobke et al., 2009 & Already included in a Cochrane Systematic Review \\
\hline
\end{tabular}




\begin{tabular}{|c|c|}
\hline Jones and Shinton, 2006 & Review article \\
\hline Jungehülsing et al., 2008 & A general population sampled \\
\hline Kasten et al., 2007 & Sample included $50 \%$ or fewer stroke patients \\
\hline Kasten et al., 2006 & Sample included $50 \%$ or fewer stroke patients \\
\hline Kerkhoff and Stögerer, 1994 & No stroke patients included \\
\hline Kim and Kim, 2005 & $\begin{array}{l}\text { Not relevant to the review - restricted to midbrain } \\
\text { stroke only }\end{array}$ \\
\hline Kissel et al., 1983 & No stroke patients included \\
\hline Klavora and Warren, 1998 & $\begin{array}{l}\text { Not relevant to the review - overview of equipment, } \\
\text { no participant data presented }\end{array}$ \\
\hline Książkiewicz and Sobczak-Kamińska, 1998 & $\begin{array}{l}\text { Not relevant to the review - eye assessment related } \\
\text { to level of consciousness }\end{array}$ \\
\hline Kumar, 2006 & News article \\
\hline Kuppersmith et al., 1996 & No stroke patients included \\
\hline Lamoreux et al., 2008 & A general population sampled \\
\hline Langelaan et al., 2007 & A general population sampled \\
\hline Leff et al., 2000 & Sample included $50 \%$ or fewer stroke patients \\
\hline Leff and Behrmann, 2008 & Review article \\
\hline Leśniak and Seniów, 2007 & Review article \\
\hline Lessell, 1975 & Review article \\
\hline Levine, 2006 & Letter to editor \\
\hline Lisabeth et al., 2009 & $\begin{array}{l}\text { Unable to distinguish with numbers of stroke and } \\
\text { TIA patients }\end{array}$ \\
\hline Macfarlane and Jolly, 1995 & Not relevant to the review - role of the orthoptist \\
\hline Markowitz, 2009 & Review article \\
\hline Marshall et al., 2008 & Not relevant to the review - fMRI study \\
\hline Marx et al., 1992 & A general population sampled \\
\hline McKean et al., 2014 & $\begin{array}{l}\text { Not relevant to the review - predicting factors on } \\
\text { imaging of stroke }\end{array}$ \\
\hline Mead et al., 2002 & Visual problems of stroke patients not discussed \\
\hline Merten, 2001 & Review article \\
\hline Mitchell et al., 1996 & A general population sampled \\
\hline Nazerian et al., 2014 & Sample included $50 \%$ or fewer stroke patients \\
\hline Nazzarko, 2007 & Review article \\
\hline Neikter, 1999 & Only conference abstract available \\
\hline Nelles et al., 2009 & $\begin{array}{l}\text { Not relevant to the review - training effects on } \\
\text { neural plasticity }\end{array}$ \\
\hline Niu et al., 2005 & $\begin{array}{l}\text { Not relevant to the review - examines location of } \\
\text { lesion for neglect }\end{array}$ \\
\hline Olbert, 1985 & Case study \\
\hline O’Neill et al., 2011 & Sample included $50 \%$ or fewer stroke patients \\
\hline Pambakian et al., 2005 & Review article \\
\hline Patel et al., 2004 & Sample included $50 \%$ or fewer stroke patients \\
\hline Patino et al., 2010 & A general population sampled \\
\hline Pelak et al., 2007 & Review article \\
\hline Peli, 2000 & Sample included $50 \%$ or fewer stroke patients \\
\hline
\end{tabular}




\begin{tabular}{|c|c|}
\hline Petzold et al., 2013 & Not related to stroke patients \\
\hline Piechocki, 2004 & News article \\
\hline Poggel et al., 2004 & Already included in a Cochrane Systematic Review \\
\hline Proto et al., 2009 & Review article \\
\hline Purvin, 1996 & Review article \\
\hline Purvin, 2004 & Review article \\
\hline Racette and Casson, 2005 & Not relevant to the review - impact on driving \\
\hline Rafałowska et al., 1972 & Only reported on three patients \\
\hline Ramrattan et al., 2001 & A general population sampled \\
\hline Riise, 1969 & No stroke patients included \\
\hline Ritchie at al., 2012 & Only reported on two patients \\
\hline Ross, 1983 & Not relevant to the review - selected sample \\
\hline Rossi et al., 1990 & Already included in a Cochrane Systematic Review \\
\hline Rowe, 2009 & Duplicate - subset sample \\
\hline Rowe, 2010 & Not relevant to the review \\
\hline Rutner et al., 2006 & Sample included $50 \%$ or fewer stroke patients \\
\hline Sabel and Kasten, 2000 & Review article \\
\hline Sabel and Mueller, 2005 & Only abstract available \\
\hline Sabel and Trauzettal-Klosinksi, 2005 & Expert debate \\
\hline Sahraie et al., 2010 & Case study \\
\hline Sand et al., 2013 & Review article \\
\hline Schofield and Leff, 2009 & Review article \\
\hline Schwartz et al., 2012 & $\begin{array}{l}\text { Not relevant to the review -assessment of eye } \\
\text { position using CT scan }\end{array}$ \\
\hline Shiraishi et al., 2004 & Stroke patients not identified separately \\
\hline Simon et al., 2003 & $\begin{array}{l}\text { Not relevant to the review -assessment of eye } \\
\text { position using CT scan }\end{array}$ \\
\hline Spitzyna et al., 2007 & Already included in a Cochrane Systematic Review \\
\hline Suchoff et al., 2008 & Sample included $50 \%$ or fewer stroke patients \\
\hline Tsai et al., 2003 & Sample included $50 \%$ or fewer stroke patients \\
\hline Unwin et al., 1999 & Only conference abstract available \\
\hline Vahlberg and Hellström, 2008 & Review article \\
\hline van der Graaff et al., 2000 & Case study \\
\hline Viken et al., 2014 & $\begin{array}{l}\text { Not relevant to the review - predicting functional } \\
\text { outcomes }\end{array}$ \\
\hline Weinberg et al., 1977 & Already included in a Cochrane Systematic Review \\
\hline Williams et al., 2003 & Visual problems not discussed \\
\hline Wolter and Preder, 2006 & Review article \\
\hline Woo and Mandelman, 1983 & Case report \\
\hline Zhang et al., 2006 & $\begin{array}{l}\text { Duplicate report of study already included in the } \\
\text { review }\end{array}$ \\
\hline Zhou et al., 2013 & A general population sampled \\
\hline Zihl, 1980 & Only reported on three patients \\
\hline Zihl et al., 1988 & Not relevant to the review \\
\hline Zihl and Hebel, 1997 & Sample included $50 \%$ or fewer stroke patients \\
\hline Zihl et al., 2009 & Not relevant to the review - selected sample \\
\hline
\end{tabular}




\section{Appendix 5. Quality Appraisal of Papers Using the STROBE Checklist}

\begin{tabular}{|c|c|c|c|c|c|c|c|c|c|c|c|c|c|c|c|c|c|c|}
\hline & 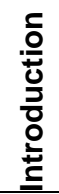 & $\begin{array}{l}\frac{0}{8} \\
0 \\
\frac{0}{0} \\
\Sigma\end{array}$ & & & & & & & & 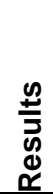 & & & & & \multicolumn{4}{|l|}{ 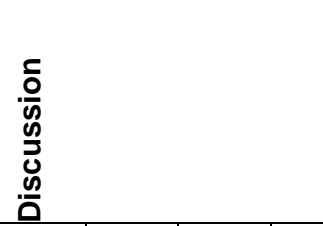 } \\
\hline & 3 & 4 & 6 & 7 & 8 & 9 & 10 & 11 & 12 & 13 & 14 & 15 & 16 & 17 & 18 & 19 & 20 & 21 \\
\hline Agrell et al., 1997 [45] & + & + & + & + & + & + & + & + & $?$ & - & + & + & + & + & + & - & + & - \\
\hline Akhtar et al., 2009 [68] & + & + & + & + & + & - & + & + & + & - & + & + & + & $\mathrm{n} / \mathrm{a}$ & + & - & - & + \\
\hline Ali et al., 2013 [30] & + & + & + & + & + & - & + & - & - & $?$ & $?$ & + & + & + & + & + & + & + \\
\hline Appelros et al., 2002 [74] & + & + & + & + & + & + & + & + & + & + & + & + & + & + & + & + & + & + \\
\hline Baier and Dieterich, 2011 [67] & - & + & + & + & + & - & - & - & - & + & + & + & + & $\mathrm{n} / \mathrm{a}$ & + & - & + & + \\
\hline Barrett et al., 2007 [38] & + & + & + & + & - & - & - & + & + & + & + & + & + & + & + & + & + & - \\
\hline Beaudoin et al., 2013 [82] & + & + & + & + & + & + & - & + & - & + & + & + & + & + & + & + & + & + \\
\hline Becker and Karnath, 2007 [76] & + & + & + & + & + & - & + & + & - & + & + & + & + & $\mathrm{n} / \mathrm{a}$ & & - & - & - \\
\hline Benedetti et al. 1993 [48] & + & + & + & + & - & - & + & + & - & + & + & + & + & + & + & - & - & + \\
\hline Bulens et al 1989 [71] & - & + & + & + & + & - & - & + & - & + & + & + & + & + & + & - & - & - \\
\hline Cassidy et al., 1998 [79] & + & + & + & + & + & - & + & + & - & - & + & + & + & $\mathrm{n} / \mathrm{a}$ & + & + & + & - \\
\hline Cassidy et al. 1999 [80] & + & + & + & + & + & - & - & + & - & + & - & + & + & $\mathrm{n} / \mathrm{a}$ & + & - & + & - \\
\hline Cassidy et al., 2001 [46] & + & + & + & + & + & + & + & + & - & + & - & + & + & + & + & + & + & - \\
\hline
\end{tabular}


Hepworth et al.; OR, 5(1): 1-43, 2016; Article no.OR.21767

\begin{tabular}{|c|c|c|c|c|c|c|c|c|c|c|c|c|c|c|c|c|c|c|}
\hline & 3 & 4 & 6 & 7 & 8 & 9 & 10 & 11 & 12 & 13 & 14 & 15 & 16 & 17 & 18 & 19 & 20 & 21 \\
\hline Celesia et al., 1997 [54] & + & + & + & + & + & - & + & + & - & + & + & + & + & + & + & - & + & - \\
\hline Chechlacz et al., 2014 [84] & - & + & - & + & + & + & + & + & + & + & + & + & + & + & + & + & + & + \\
\hline Clisby, 1995 [32] & + & - & - & - & - & - & - & - & - & + & - & + & - & - & - & - & - & - \\
\hline De Renzi et al., 1982 [62] & - & + & + & + & + & + & + & + & - & + & + & + & + & $\mathrm{n} / \mathrm{a}$ & + & - & + & - \\
\hline Dos Santos et al., 2012 [72] & + & - & + & + & + & + & - & + & + & + & + & + & + & $\mathrm{n} / \mathrm{a}$ & + & + & + & + \\
\hline Edwards et al., 2006 [70] & + & + & + & + & + & + & - & + & + & + & + & + & + & + & + & - & + & + \\
\hline Farné et al., 2004 [89] & + & + & - & + & + & - & - & + & + & + & + & + & + & + & + & - & - & - \\
\hline Fowler et al., 1996 [58] & + & + & + & + & + & - & + & + & - & + & + & + & + & $\mathrm{n} / \mathrm{a}$ & + & - & + & - \\
\hline Freeman and Rudge, 1987 [35] & + & + & + & + & + & - & + & + & - & + & + & + & + & $\mathrm{n} / \mathrm{a}$ & + & - & - & - \\
\hline Gall et al., 2010 [31] & + & + & + & + & + & + & + & + & + & + & + & + & + & $\mathrm{n} / \mathrm{a}$ & + & + & + & + \\
\hline Gray et al., 1989 [41] & + & + & + & + & + & + & + & + & - & + & + & + & + & $\mathrm{n} / \mathrm{a}$ & + & - & - & - \\
\hline Haerer, 1973 [47] & + & + & + & + & - & - & + & - & - & + & + & + & + & $\mathrm{n} / \mathrm{a}$ & + & + & + & - \\
\hline Isaeff et al., 1974 [33] & - & + & $?$ & - & - & - & - & + & - & + & + & + & + & $\mathrm{n} / \mathrm{a}$ & - & - & - & - \\
\hline Jerath et al., 2011 [53] & + & + & + & + & + & - & + & + & - & + & + & + & + & $\mathrm{n} / \mathrm{a}$ & + & + & + & + \\
\hline Kedar et al., 2007 [55] & + & + & + & + & + & + & + & + & + & + & + & + & + & + & + & + & + & - \\
\hline Lawrence et al., 2001 [49] & + & + & + & + & - & - & + & + & + & + & + & + & + & + & + & - & - & - \\
\hline
\end{tabular}


Hepworth et al.; OR, 5(1): 1-43, 2016; Article no.OR.21767

\begin{tabular}{|c|c|c|c|c|c|c|c|c|c|c|c|c|c|c|c|c|c|c|}
\hline & 3 & 4 & 6 & 7 & 8 & 9 & 10 & 11 & 12 & 13 & 14 & 15 & 16 & 17 & 18 & 19 & 20 & 21 \\
\hline Lee et al., 2009 [78] & - & + & + & + & + & - & + & + & - & + & + & + & + & + & + & - & + & - \\
\hline Linden et al., 2006 [75] & + & + & + & + & + & - & + & + & + & + & + & + & + & $\mathrm{n} / \mathrm{a}$ & + & - & + & - \\
\hline Lotery et al., 2000 [64] & + & + & + & + & - & - & - & + & - & + & + & + & + & $\mathrm{n} / \mathrm{a}$ & + & - & - & - \\
\hline Maeshima et al., 2012 [59] & - & + & + & + & + & - & + & + & + & + & + & + & + & $\mathrm{n} / \mathrm{a}$ & + & - & - & - \\
\hline $\mathrm{Ng}$ et al., 2005 [50] & + & + & + & + & + & - & + & + & + & + & + & + & + & + & + & + & + & + \\
\hline Pedersen et al., 1997 [73] & + & + & + & + & + & - & + & + & + & + & + & + & + & + & + & - & - & - \\
\hline Poggel et al., 2007 [89] & - & + & + & + & + & - & + & + & + & + & + & + & + & + & + & - & - & - \\
\hline Rathore et al., 2002 [52] & - & - & + & + & - & - & + & + & + & + & + & + & + & + & + & + & + & - \\
\hline Rowe, 2007 [69] & + & + & + & + & - & - & + & + & + & + & + & + & + & + & + & - & + & + \\
\hline Rowe et al., 2008 [66] & + & + & + & + & - & - & + & + & - & + & + & + & + & + & + & - & + & + \\
\hline Rowe et al., 2009 [24] & + & + & + & + & - & - & + & + & - & + & + & + & + & + & + & - & + & + \\
\hline Rowe et al., 2009 [6] & + & + & + & + & + & - & + & + & - & + & + & + & + & + & + & + & + & + \\
\hline Rowe et al., 2010 [23] & + & + & + & + & - & - & + & + & - & + & + & + & + & + & + & - & + & - \\
\hline Rowe et al., 2011 [18] & + & + & + & + & + & - & + & + & + & + & + & + & + & + & + & + & + & + \\
\hline Rowe et al., 2011 [19] & + & + & + & + & + & - & + & + & + & + & + & + & + & + & + & + & + & - \\
\hline Rowe et al., 2013 [36] & + & + & + & + & + & - & + & + & + & + & + & + & + & + & + & - & + & - \\
\hline
\end{tabular}


Hepworth et al.; OR, 5(1): 1-43, 2016; Article no.OR.21767

\begin{tabular}{|c|c|c|c|c|c|c|c|c|c|c|c|c|c|c|c|c|c|c|}
\hline & 3 & 4 & 6 & 7 & 8 & 9 & 10 & 11 & 12 & 13 & 14 & 15 & 16 & 17 & 18 & 19 & 20 & 21 \\
\hline Rowe et al., 2013 [22] & + & + & + & + & - & 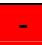 & + & + & + & + & + & + & + & + & + & + & + & - \\
\hline Rowe et al., 2013 [21] & + & + & + & + & + & - & + & + & + & + & + & + & + & + & + & + & + & + \\
\hline Schmielau and Wong Jr, 2007 [86] & + & - & + & + & + & - & - & + & - & + & + & + & + & + & + & - & - & - \\
\hline Searls et al., 2012 [42] & + & + & + & + & - & - & + & + & + & + & + & + & + & + & + & - & - & - \\
\hline Shrestha et al., 2012 [81] & + & + & + & + & + & - & + & + & + & + & + & + & + & + & + & - & - & - \\
\hline Siddique et al., 2009 [65] & - & + & + & - & - & - & + & + & - & + & + & + & + & + & + & - & + & + \\
\hline Singer et al., 2006 [63] & - & + & + & + & - & - & + & + & + & + & + & + & + & + & + & + & + & + \\
\hline Siong et al., 2014 [61] & + & + & + & + & + & + & + & + & + & + & + & + & + & + & + & + & + & + \\
\hline Stone et al., 1993 [56] & + & + & + & + & + & - & + & + & + & + & + & + & + & + & + & - & - & - \\
\hline Su and Young, 2013 [60] & - & + & + & + & + & - & + & + & - & + & + & + & - & - & + & - & + & - \\
\hline Tao et al., 2012 [43] & + & + & + & + & - & - & + & + & + & + & + & + & + & + & + & + & + & - \\
\hline Tiel and Kölmel, 1991 [85] & - & + & + & + & - & - & + & + & - & + & + & + & - & $\mathrm{n} / \mathrm{a}$ & + & - & - & - \\
\hline Townsend et al., 2007 [51] & - & + & + & + & + & + & + & + & + & + & + & + & + & $\mathrm{n} / \mathrm{a}$ & + & - & - & + \\
\hline Trobe et al., 1973 [34] & - & + & + & - & - & - & + & - & - & + & + & + & - & - & + & - & - & - \\
\hline van Nes et al., 2009 [77] & + & + & + & + & + & + & + & + & + & + & + & + & + & + & + & - & - & - \\
\hline Yang et al., 2014 [83] & + & + & + & + & + & + & + & + & + & + & + & + & + & + & + & - & - & - \\
\hline
\end{tabular}


Hepworth et al.; OR, 5(1): 1-43, 2016; Article no.OR.21767

\begin{tabular}{|l|c|c|c|c|c|c|c|c|c|c|c|c|c|c|c|c|c|c|}
\hline & 3 & 4 & 6 & 7 & 8 & 9 & 10 & 11 & 12 & 13 & 14 & 15 & 16 & 17 & 18 & 19 & 20 & 21 \\
\hline Yap et al., 1975 [57] & + & + & + & + & - & - & + & + & - & + & + & + & - & $\mathrm{n} / \mathrm{a}$ & + & - & - & - \\
\hline Zhang et al., 2006a [44] & + & + & + & + & + & + & + & + & - & + & + & + & + & + & + & - \\
\hline
\end{tabular}

(9) 2016 Hepworth et al.; This is an Open Access article distributed under the terms of the Creative Commons Attribution License (http://creativecommons.org/licenses/by/4.0), which permits unrestricted use, distribution, and reproduction in any medium, provided the original work is properly cited.

\section{Peer-review history:}

The peer review history for this paper can be accessed here:

http://sciencedomain.org/review-history/12294 